\title{
Quantum heat engine with coupled superconducting resonators
}

\author{
Ali Ü. C. Hardal, ${ }^{1,2}$ Nur Aslan, ${ }^{1}$ C. M. Wilson, ${ }^{3}$ and Özgür E. Müstecaplıoğlu ${ }^{1, *}$ \\ ${ }^{1}$ Department of Physics, Koç University, Sartyer, Istanbul, 34450, Turkey \\ ${ }^{2}$ Department of Photonics Engineering, Technical University of Denmark, Ørsteds Plads 343, DK-2800 Kgs. Lyngby, Denmark \\ ${ }^{3}$ Institute of Quantum Computing and Electrical and Computer Engineering, University of Waterloo, Waterloo, Ontario, Canada N2L 3G1
}

(Received 14 August 2017; published 18 December 2017)

\begin{abstract}
We propose a quantum heat engine composed of two superconducting transmission line resonators interacting with each other via an optomechanical-like coupling. One resonator is periodically excited by a thermal pump. The incoherently driven resonator induces coherent oscillations in the other one due to the coupling. A limit cycle, indicating finite power output, emerges in the thermodynamical phase space. The system implements an all-electrical analog of a photonic piston. Instead of mechanical motion, the power output is obtained as a coherent electrical charging in our case. We explore the differences between the quantum and classical descriptions of our system by solving the quantum master equation and classical Langevin equations. Specifically, we calculate the mean number of excitations, second-order coherence, as well as the entropy, temperature, power, and mean energy to reveal the signatures of quantum behavior in the statistical and thermodynamic properties of the system. We find evidence of a quantum enhancement in the power output of the engine at low temperatures.
\end{abstract}

DOI: 10.1103/PhysRevE.96.062120

\section{INTRODUCTION}

Heat engines with quantum working substances, so-called quantum heat engines (QHEs), have attracted much attention recently [1-39]. The steam driven mechanical piston is an archetype of classical heat engines. Quantum analogs of piston engines have been proposed using optomechanical models, where steam is replaced by a photonic gas [24-29]. A single-atom piston engine described by an effective optomechanical model has been demonstrated in the classical regime very recently [40]. The benefits of "quantumness" $[41,42]$ as well as the quantum-to-classical transition [43] in heat engines are fundamental problems in the emerging field of quantum thermodynamics. We propose a quantum heat engine composed of a pair of superconducting resonators interacting via an effective optomechanical coupling [44]. It offers an on-chip circuit analog of a piston engine and could be used to explore fundamental quantum properties in heat engines.

We specifically consider a system of two coupled superconducting resonators [44] as an alternative embodiment of the piston cycle used in the single-atom heat engine [40]. In our case, which is shown in Fig. 1, the resonator modes play the role of vibrational modes of the trapped atom. One of the resonators is periodically driven by a quasithermal pump and interacts with the other resonator through an effective optomechanical coupling [44]. The emergence of coherence in one mode by incoherent excitation of the other is a typical feature of quantum piston engines exploiting optomechanical coupling [27]. We investigate the quantum statistics of the resonator modes by calculating the mean number dynamics, occupation probability distributions, and second-order correlation functions. We verify that the incoherently driven mode remains thermal while the other becomes almost coherent. In addition, thermodynamic properties of the system are examined by calculating the mean energy versus effective electrical length of the driven resonator and the temperature versus entropy diagrams of the engine. We identify that the system

\footnotetext{
*omustecap@ku.edu.tr
}

undergoes an Otto engine cycle. We find that, after a transient regime, a limit cycle emerges in the thermodynamical phase space, indicating finite power output, at the same time that the driven mode induces steady coherent oscillations in the other mode. Quantum coherence in the energy basis of the total system can be formally related to the inner quantum friction and irreversible work [45]. In our case quantum coherence is in one of the resonator modes storing the work output.

Further, we compare the quantum mechanical results with those obtained from the classical Langevin equations of motion. We find significant qualitative and quantitative differences between the classical and quantum descriptions of the system. In particular, the power output from the quantum piston engine is greater than that of the classical one at low temperatures. We argue that the quantum enhancement in power output can be observed in superconducting resonators. We explain the effect by identifying the type of quantum correlation behind the power output as the so-called signalmeter correlation $[46,47]$ and compare it with the classical correlations. It is not a universal fact that quantum correlations can always increase the power output of a piston engine. Recent studies reported exactly the opposite conclusions where quantum fluctuations cause less power output in a rotor type piston engine [35].

This paper is organized as follows. In Sec. II, we introduce the quantum optomechanical engine model based on coupled superconducting transmission line resonators. In Sec. III, we describe our numerical methods and present the results of quantum dynamical simulations. The corresponding classical engine model is introduced in Sec. IV and the results of the classical Langevin equation simulations are given in Sec. V. These results are compared to the quantum description. In Sec. VI, we discuss the quantum character of the model system in terms of the quantum coherence and correlations. We conclude in Sec. VII.

\section{THE QUANTUM MODEL}

Our model system consists of two superconducting transmission line resonators on a chip, held at temperature $T_{0}$, 


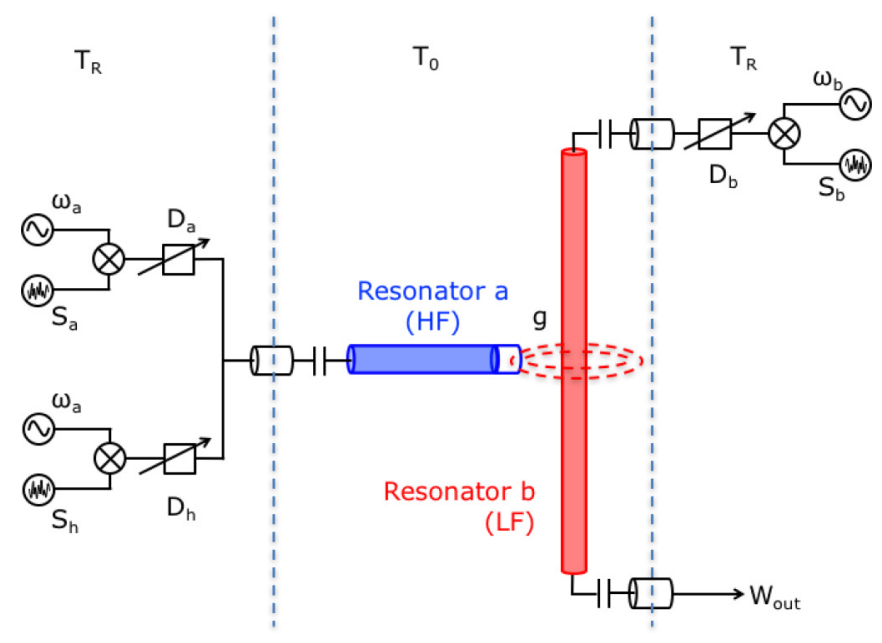

FIG. 1. Circuit diagram of the quantum heat engine composed of two superconducting microwave resonators interacting via an effective optomechanical like coupling with strength $g$. Resonators indicated by "a" and "b" have high-frequency (HF) and low-frequency (LF) modes with $\omega_{a} \gg \omega_{b}$. The HF mode is periodically driven by a variable amplitude attenuation $\left(D_{h}\right)$ white noise source $\left(S_{h}\right)$, corresponding to an effective hot bath at $T_{h}$. The resonators are on a chip at a background temperature $T_{0}$. External noise drives are at room temperature $T_{R}$. Both the LF and HF resonators are subject to additional quasithermal sources implemented by amplitude controlled $\left(D_{a}, D_{b}\right)$ white noise drives $\left(S_{a}, S_{b}\right)$, which are constantly applied to the corresponding resonators.

as shown in Fig. 1. A resonator, indicated by "a" in the figure, is terminated by a SQUID, which collects the flux generated by the other resonator, indicated by "b," so that an effective optomechanical-like coupling, denoted by $g$, between the resonators can be engineered [44]. We assume resonator " $a$ " with frequency $\omega_{a}$ is shorter than resonator " $b$ " with frequency $\omega_{b}$ such that $\omega_{a} \gg \omega_{b}$. The high-frequency (HF) and low-frequency (LF) modes of resonator "a" and resonator "b" can be considered as analogs of the optical and mechanical modes of an optomechanical system, respectively. The effective optomechanical-like coupling between the resonators is expressed as (we take $\hbar=1$ ) [44]

$$
\hat{H}_{\mathrm{sys}}=\omega_{a} \hat{a}^{\dagger} \hat{a}+\omega_{b} \hat{b}^{\dagger} b-g \hat{a}^{\dagger} \hat{a}\left(\hat{b}+\hat{b}^{\dagger}\right),
$$

where $\hat{a}\left(\hat{a}^{\dagger}\right)$ and $\hat{b}\left(\hat{b}^{\dagger}\right)$ are the annihilation (creation) operators for the HF and LF modes, respectively.

In addition to the cold environment at $T_{0}$, we assume three microwave white noise drives are applied to the resonators. These drives are produced by external sources at room temperature, $T_{R}$. We consider two continuously applied noises with power spectral densities $S_{a}$ and $S_{b}$ on the resonators a and $b$, respectively. Their amplitudes can be controlled by variable amplitude attenuators $D_{a}$ and $D_{b}$. The HF resonator is subject to another amplitude-controlled $\left(D_{h}\right)$ and periodic white noise source with a power spectral density $S_{h}$. The power spectral densities are assumed to be narrow band, centered at the corresponding resonator frequencies $\omega_{a}$ and $\omega_{b}$, but much wider than the bandwidth of the HF resonator. Accordingly, each externally applied noise source approximates a one-dimensional black body (thermal) spectrum [48] at effective temperatures that can be determined from the Planck distribution functions (we take $k_{B}=1$ ),

$$
\begin{aligned}
\bar{n}_{a} & =\frac{1}{\exp \left(\omega_{a} / T_{a}\right)-1}, \\
\bar{n}_{b} & =\frac{1}{\exp \left(\omega_{b} / T_{b}\right)-1}, \\
\bar{n}_{h} & =\frac{1}{\exp \left(\omega_{a} / T_{h}\right)-1},
\end{aligned}
$$

where $T_{h}, T_{b}$, and $T_{a}$ are the effective temperatures corresponding to the periodic drive, continuous drive on the $\mathrm{HF}$ resonator, and the continuous drive on the LF resonator, respectively. We assume the periodic drive is used to engineer an effective hot bath such that $T_{h}>T_{a}, T_{b}$. The mean number of excitations in the cold baths are denoted by $\bar{n}_{a}$ and $\bar{n}_{b}$ for the HF and LF modes, respectively. The mean number of excitations in the periodically modulated hot reservoir is denoted by $\bar{n}_{h}$. The one-dimensional Planck's law gives the power spectral densities as $S_{x}=\omega_{x} \bar{n}_{x}$ with $x=a, b, h$. We consider engineering the two additional cold baths to get more flexibility to reach desired steady states in the engine operation, which may not be achieved in the case of a common single environment at $T_{0}$. A similar strategy was employed for the case of the single-atom piston engine by using an additional cooling laser [40].

The dynamics of the density matrix $\hat{\rho}$ of the resonator pair can be determined by a master equation [48],

$$
\begin{aligned}
\dot{\hat{\rho}}= & -i\left[\hat{H}_{\mathrm{sys}}, \hat{\rho}\right]+\kappa_{a}\left(\bar{n}_{a}+1\right) D[\hat{a}]+\kappa_{a} \bar{n}_{a} D\left[\hat{a}^{\dagger}\right] \\
& +\kappa_{b}\left(\bar{n}_{b}+1\right) D[\hat{b}]+\kappa_{b} \bar{n}_{b} D\left[\hat{b}^{\dagger}\right] \\
& +\kappa_{h}(t)\left(\bar{n}_{h}+1\right) D[\hat{a}]+\kappa_{h}(t) \bar{n}_{h} D\left[\hat{a}^{\dagger}\right] \\
& +\kappa_{0}^{a}\left(\bar{n}_{0}^{a}+1\right) D[\hat{a}]+\kappa_{0}^{a} \bar{n}_{0}^{a} D\left[\hat{a}^{\dagger}\right] \\
& +\kappa_{0}^{b}\left(\bar{n}_{0}^{b}+1\right) D[\hat{b}]+\kappa_{0}^{b} \bar{n}_{0}^{b} D\left[\hat{b}^{\dagger}\right],
\end{aligned}
$$

where $D[\hat{\alpha}]:=(1 / 2)\left(2 \hat{\alpha} \hat{\rho} \hat{\alpha}^{\dagger}-\hat{\alpha}^{\dagger} \hat{\alpha} \hat{\rho}-\hat{\rho} \hat{\alpha}^{\dagger} \hat{\alpha}\right)$ refers to the Lindblad dissipator superoperators with $\hat{\alpha}=\hat{a}, \hat{b} . \kappa_{a}$ and $\kappa_{b}$ are effective coupling constants of the HF and LF modes with their local cold baths, respectively. The coupling coefficient of the HF mode with the effective hot bath is denoted by $\kappa_{h}(t)$, which has a periodic time dependence. Small background field excitations are denoted by $n_{0}^{a}$ and $n_{0}^{b}$ in the corresponding terms of the master equation describing the coupling of the environment at $T_{0}$ with the HF and LF resonators at rates $\kappa_{0}^{a}$ and $\kappa_{0}^{a}$, respectively.

We assume a special case that each local cold bath, engineered with the quasithermal noise drive, has the same excitation number so that we can introduce $\bar{n}_{c}:=\bar{n}_{a}=\bar{n}_{b}$, which is possible for $T_{a} / T_{b}=\omega_{a} / \omega_{b}$. Accordingly, we have $T_{a} \gg T_{b}$. It may be worth emphasizing that the local temperatures are "effective" and can be high (e.g., $100 \mathrm{~K}$ or more), without affecting superconductivity, because very little of the noise power is absorbed by the resonators [48].

The master equation we consider assumes the usual BornMarkov approximations under the weak coupling with the noise sources. Moreover, as we use local effective reservoirs in the dissipators, it is not immediately obvious if the dynamics are consistent with the second law of thermodynamics at all parameter regimes [49-51]. It is usually assumed that such a 
local master equation should be reliable when the coupling between the subsystems (here the HF and LF resonators) is sufficiently weak $[52,53]$. The local master equation may still be applicable even for relatively strong coupling if the environmental noise has sufficiently wide bandwidth. Our scheme involves only the thermal white noises, with relatively small effective temperatures with respect to the resonator frequencies. Hence we expect the validity of the local approach [52,54].

To determine the validity regime more strictly for our case, we also used a "global" master equation (see Appendix A) derived for arbitrary optomechanical coupling strengths [55] to compare the results with the "local" master equation. We found that both local and global master equation results agree well in the range under consideration $g \leqslant \omega_{b}$. The regime of $g=\omega_{b}$ is not exactly the ultrastrong coupling regime of optomechanics, as we have large $\kappa_{a}>g$. In the temperature regimes we consider, the excitation of the HF resonator is weak $\left\langle n_{a}\right\rangle<1$. We explore a regime of single "photon" optomechanics which is not well charted.

\section{QUANTUM DYNAMICS OF THE ENGINE}

In our simulations we use dimensionless parameters by scaling $\omega_{b} / 2 \pi=500 \mathrm{MHz}, g / 2 \pi=500 \mathrm{MHz}, \kappa_{h} / 2 \pi=$ $\kappa_{a} / 2 \pi=2 \mathrm{GHz}$, and $\kappa_{b} / 2 \pi=50 \mathrm{MHz}$ by $\omega_{a} / 2 \pi=10 \mathrm{GHz}$. The temporal profile of the incoherent drive acting on the HF mode is taken as a square wave $\kappa_{h}(t):=\kappa_{h} s(t)$. The square wave $s(t)=1$ and $s(t)=0$ for the heating and cooling stages, respectively. Each stage takes the same time of $\pi / \omega_{b}$. In units of $1 / \omega_{a}$ the cycle duration is then $t_{\text {cycle }}=(2 \pi) 20$ (cf. Fig. 2 ).

The mean number of excitations in the effective baths are taken to be $\bar{n}_{a}=\bar{n}_{b}:=\bar{n}_{c}=0.01$ and $\bar{n}_{h}>0.1$, for which our simulations yield limit cycles for the engine operation at steady state. This choice of identical thermal occupation numbers is only a convenience in numerical simulations to reduce the number of control parameters; it is not essential for the engine operation. The corresponding effective temperatures become $T_{a} \sim 104 \mathrm{mK}$ and $T_{b} \sim 5 \mathrm{mK}$. A typical environment temperature for the superconducting resonators is $T_{0} \sim 20 \mathrm{mK}$. Accordingly the hierarchy of temperatures associated with the engineered environments for the resonators becomes $T_{h}>T_{a}>T_{0}>T_{b}$. The temperature ranges of $T_{h}>$ $T_{a}>T_{0}$ have been successfully produced experimentally for a single superconducting resonator using the noise drive method [48]. The range of $T_{0}>T_{b}$ can be engineered using proposals for optomechanical schemes [56]. The HF resonator mode is effectively heated to $T_{h}$ when the external periodic noise pulse is on, and when the pulse is off it effectively cools to $T_{a}$. The LF resonator is always coupled to an effective cold bath.

The quantum dynamics of the coupled resonators subject to such effective heating and cooling stages is investigated by solving Eq. (5) using QuTiP [57]. We neglect the last four terms associated with the background environment in Eq. (5) by assuming $\kappa_{a} \gg \kappa_{0}^{a}$ and $\kappa_{0}^{b} \bar{n}_{0}^{b}>\kappa_{b} \bar{n}_{b}$. After an intake stage, where the resonators are at their respective initial states, repeated action of the the heating and cooling stages will lead the system into a limit cycle that can be considered as a heat engine cycle with a net power output.

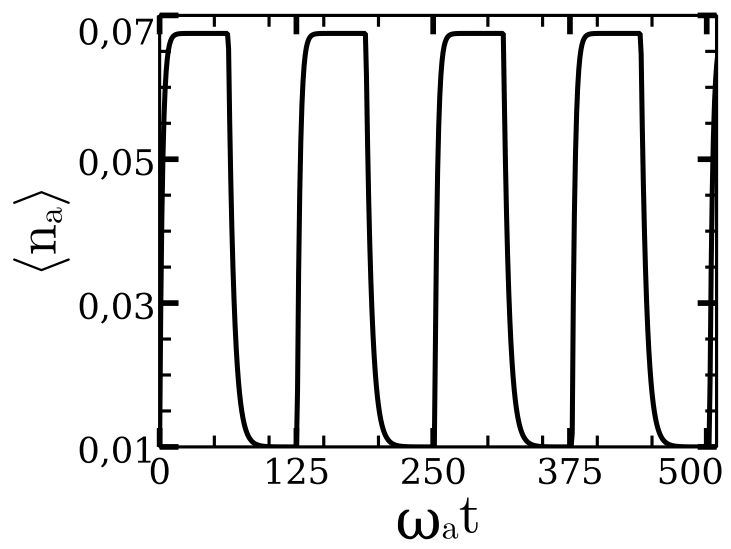

(a)

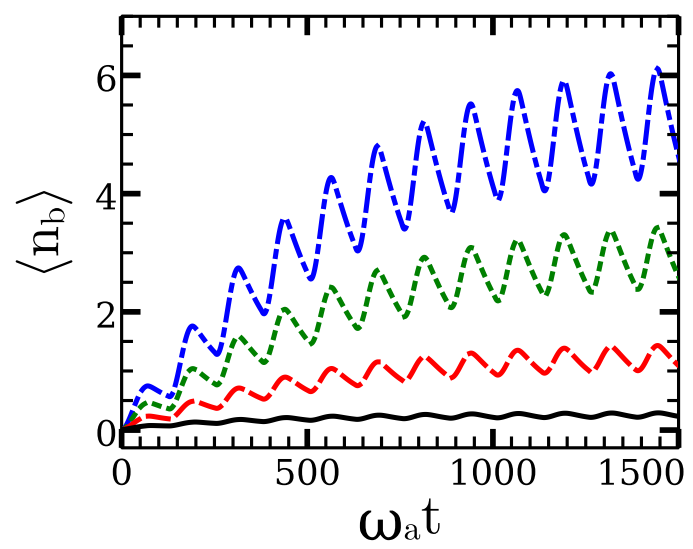

(b)

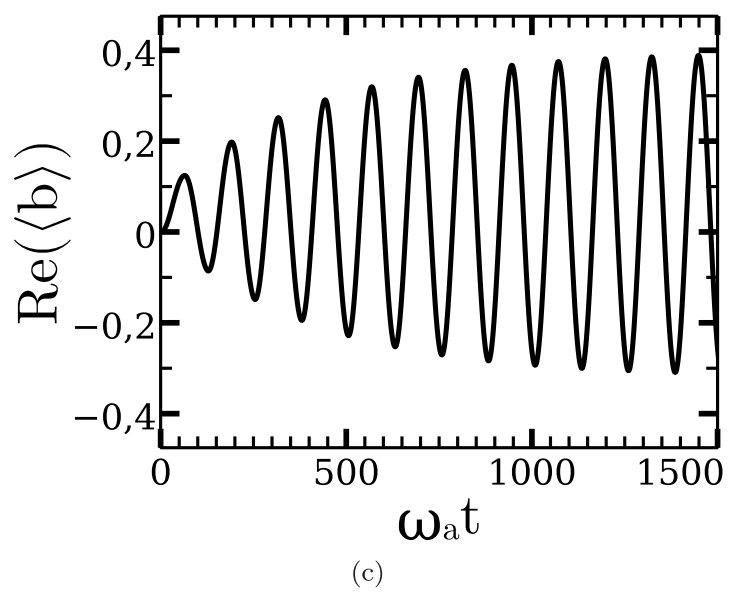

FIG. 2. Dynamics of the mean number of excitations (a) $\left\langle\hat{n}_{a}\right\rangle$ and (b) $\left\langle\hat{n}_{b}\right\rangle$ in the HF and LF modes, respectively, for $\bar{n}_{h}=0.125$ (black solid), $\bar{n}_{h}=0.325$ (red dashed), $\bar{n}_{h}=0.525$ (green dotted), and $\bar{n}_{h}=$ 0.725 (blue dot-dashed). (c) Dynamics of the LF field coherence is characterized by $\Re(\langle b\rangle)$, where $\Re \equiv$ Re denotes the real part. Time is dimensionless and scaled with the HF mode frequency $\omega_{a}$. All the other parameters are as explained in the text.

The system is assumed to be in an initial state $\hat{\rho}(0)=$ $\hat{\rho}_{a}(0) \otimes \hat{\rho}_{b}(0)$, where

$$
\begin{aligned}
& \hat{\rho}_{a}(0)=\frac{1}{\operatorname{Tr} e^{-\omega_{a} \hat{a}^{\dagger} \hat{a} / T_{a}}} e^{-\omega_{a} \hat{a}^{\dagger} \hat{a} / T_{a}}, \\
& \hat{\rho}_{b}(0)=\frac{1}{\operatorname{Tr} e^{-\omega_{b} \hat{b} \hat{\dagger} \hat{b} / T_{b}}} e^{-\omega_{b} \hat{b}^{\dagger} \hat{b} / T_{b}},
\end{aligned}
$$


are the initial density matrix operators of the HF and LF modes, respectively. Initial occupations of the modes then are $\bar{n}_{c}$ since $\left\langle\hat{n}_{x}\right\rangle(0)=\left\langle\hat{x}^{\dagger} \hat{x}\right\rangle(0)=\operatorname{Tr}\left(\hat{\rho}(0) \hat{x}^{\dagger} \hat{x}\right)=\bar{n}_{c}=0.01$, with $x=a, b$.

\section{A. Quantum piston in phase space}

The heating and cooling stages in the engine operation of the coupled resonator system can be visualized by examining the dynamics of the mean excitation numbers $\left\langle\hat{n}_{x}\right\rangle=\left\langle\hat{x}^{\dagger} \hat{x}\right\rangle=$ $\operatorname{Tr}\left(\hat{\rho}(t) \hat{x}^{\dagger} \hat{x}\right)$, with $x=a, b$, which are shown in Fig. 2 . The dynamics of $\left\langle\hat{n}_{a}\right\rangle$ is shown in Fig. 2(a) for $\bar{n}_{h}=0.125$. The heating pulse duration is taken to be longer than the thermalization time of the HF resonator. When the heating pulse is applied, the HF mode reaches a steady state at $\left\langle\hat{n}_{a}\right\rangle_{\mathrm{ss}} \sim 0.0675$, which is the same as the analytical result $\left\langle\hat{n}_{a}\right\rangle_{\mathrm{ss}}=\left(\bar{n}_{c}+\bar{n}_{h}\right) / 2$ (see Appendix B). After the heating pulse, the excitation number first rapidly drops and then slowly cools back to the initial value. Analytical expressions can be approximately given as

$$
\left\langle\hat{n}_{a}\right\rangle= \begin{cases}\frac{\bar{n}_{a}+\bar{n}_{h}}{2}-\frac{\bar{n}_{h}-\bar{n}_{a}}{2} e^{-\left(\kappa_{a}+\kappa_{h}\right) t}, & 0 \leqslant t<\frac{\pi}{\omega_{b}} ; \\ \bar{n}_{a}+\frac{\bar{n}_{h}-\bar{n}_{a}}{2} e^{-\kappa_{a}\left(t-\pi / \omega_{b}\right)}, & \frac{\pi}{\omega_{b}} \leqslant t<\frac{2 \pi}{\omega_{b}},\end{cases}
$$

which is repeated indefinitely.

The dynamics of the mean excitations of the LF mode $\left\langle\hat{n}_{b}\right\rangle$ are shown in Fig. 2(b), which reaches a steady state with coherent oscillations in the long-time limit. Both the time average $\left\langle\hat{n}_{b}\right\rangle$ value and the amplitude of the oscillations are larger for larger $\bar{n}_{h}$, while the frequency of oscillations remains the same. The heating pulse duration is only long enough to thermalize the HF resonator, while the LF resonator is unable to equilibrate in a pulse period. The evolution of the LF resonator will start from a different initial condition for every pulse action. This "memory" effect leads to a higher time average $\left\langle\hat{n}_{b}\right\rangle$ value than the one found under continuous drive (cf. Appendix B). In addition, correlations between the HF and LF resonator modes contribute to to the dynamics of the LF mode excitations. We will explore the physical mechanisms behind the dynamics of $\left\langle n_{b}\right\rangle$ in more detail in Sec. VI.

Figure 2(c) plots the "electrical displacement" of the LF mode, $\mathfrak{R}(\langle\hat{b}\rangle)=q / 2$, where $q=\langle\hat{q}\rangle=\left\langle\hat{b}+\hat{b}^{\dagger}\right\rangle$, showing coherent oscillations in the steady state. The notation $\Re(\cdot)$ stands for the real part. In contrast to the dynamics of $\left\langle n_{b}\right\rangle, q$ has identical classical and quantum dynamics. Its equation of motion (see the Appendix A and C) is that of a periodically driven damped oscillator, which is given by

$$
\ddot{q}+\kappa_{b} \dot{q}+\omega^{2} q=2 \omega_{b} g\left\langle n_{a}(t)\right\rangle,
$$

where $\omega^{2}=\omega_{b}^{2}+\kappa_{b}^{2} / 4$ and the dots over $q$ indicate time derivatives. This equation can be interpreted in terms of an effective series Resistor-Inductor-Capacitor (RLC) circuit. The cold environment of the LF resonator acts as the resistive element dampening the LC oscillations. In the standard way, the natural frequency $\omega_{b}$ of the LC oscillations is further renormalized by the damping rate $\kappa_{b}$. The $\mathrm{HF}$ resonator provides the input voltage which sustains the oscillations. Despite the thermal nature of the HF resonator, it drives the RLC circuit into coherent oscillations because the drive only depends on $\left\langle n_{a}\right\rangle$, which is alternating between high and low values periodically. We note that while $q$ is not vanishing in the steady state, the electrical momentum $p=\langle\hat{p}\rangle=i\left\langle\left(\hat{b}^{\dagger}-\hat{b}\right)\right\rangle$ becomes approximately zero (It is exactly zero according to the global master equation as can be seen in Appendix B. However, this may be an unphysical artifact of the secular approximation in the global approach which may predict coherences out of equilibrium inaccurately in some cases [58]).

The LF oscillator is in the weak damping regime $\left(\kappa_{b}<\omega_{b}\right)$. The formal solution of Eq. (9) is given by

$$
q(t)=2 g \int_{0}^{t} d t^{\prime}\left\langle n_{a}\left(t^{\prime}\right)\right\rangle e^{-\frac{\kappa_{b}}{2}\left(t-t^{\prime}\right)} \sin \left[\omega_{b}\left(t-t^{\prime}\right)\right] .
$$

The periodic nature of $\left\langle n_{a}\left(t^{\prime}\right)\right\rangle$ leads to an intuitive understanding of the emergence of a coherent steady state in $q(t)$. Harmonics of $\left\langle n_{a}\left(t^{\prime}\right)\right\rangle$ are given by the frequencies $\omega_{k}=k \omega_{b}$ where $k$ is an integer. According to Eq. (10) the maximum overlap or the resonance would occur for the first harmonic $k=1$. Besides, the higher harmonics would have relatively smaller significance as their amplitudes get smaller with $k$. Accordingly, steady-state oscillations in $q(t)$ are dominated by the single frequency $\omega_{b}$. The time average value of $q$, as well as the amplitude of oscillations, increase linearly with $\bar{n}_{h}$. In terms of our physical parameters, the approximate analytical solution is found to be

$$
q=\frac{\bar{n}_{h}+3 \bar{n}_{c}}{2}+\frac{20\left(\bar{n}_{h}-\bar{n}_{c}\right)}{\pi} \sin \left(\omega_{b} t-1.55\right) .
$$

A more general solution is given in Appendix C.

We further visualize the coherence in the steady state of the LF mode by investigating the probability distribution $P(n):=$ $\left\langle n\left|\rho_{b}(t)\right| n\right\rangle$, where $\rho_{b}(t)=\operatorname{Tr}_{a}[\rho(t)]$ is the reduced density matrix of the LF mode, in Fig. 3 . As we increase $\bar{n}_{h}$ from $\bar{n}_{h}=$ 0.125 to $\bar{n}_{h}=0.725$, the the probability distribution changes from a thermal distribution to a coherent distribution, as shown in Figs. 3(a)-3(d).

In Fig. 4, we plot the dynamics of the mean values of the field quadratures $q_{b}:=\left\langle\hat{q}_{\boldsymbol{b}}\right\rangle:=q / \sqrt{2}$ and $p_{b}:=\left\langle\hat{p}_{\boldsymbol{b}}\right\rangle:=$ $p / \sqrt{2}$, where $p=\langle\hat{p}\rangle:=i\left\langle\left(\hat{b}^{\dagger}-\hat{b}\right)\right\rangle$, with respect to each other. The resulting phase diagram is that of a periodically driven damped displaced harmonic oscillator, where the slight shift of the center of the limit cycle from the origin is the signature of the coherent displacement induced by the thermal drive. The HF mode reaches steady state within a single thermal noise pulse duration as shown in Fig. 2(a). The action of the thermal noise pulse on the HF mode is translated to the LF mode by the optomechanical coupling. According to Fig. 2(b), the LF mode can reach an oscillatory steady state after the action of a several noise pulses. As the LF mode lags behind the HF mode, there will be a transient regime before a limit cycle is established, as we see in Fig. 4. While a limit cycle emerges for our model, we note that the existence of a stable limit cycle after transients is in general model and initial condition dependent [59]. Within the approximation of $\left\langle\hat{n}_{a}\right\rangle$ as a square wave drive, we find an expression for the limit cycle (see Appendix C)

$$
\left(p_{b}-p_{b 0}\right)^{2}+\left(q_{b}-q_{b 0}\right)^{2}=R^{2}
$$




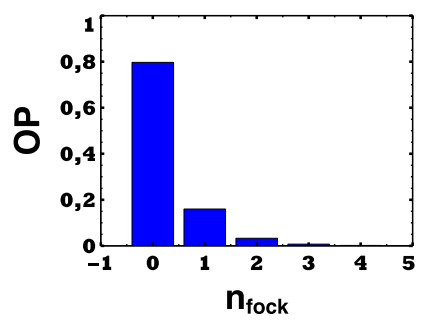

(a)

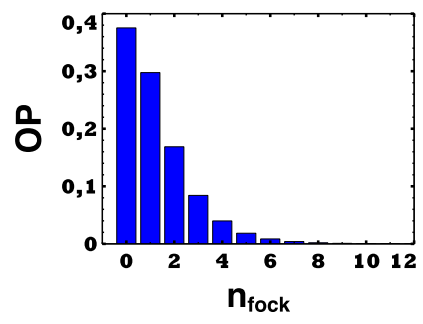

(b)

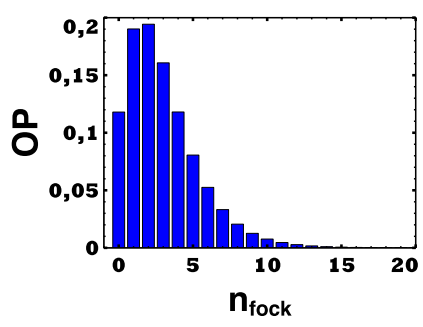

(c)

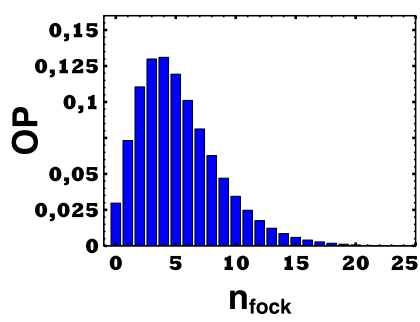

(d)

FIG. 3. [(a)-(d)] The occupation probability (OP) with respect to occupation number of the Fock states $n_{\text {fock }}$ of the LF resonator mode at $\omega_{a} t=5000$ for $\bar{n}_{h}=0.125$ (a), $\bar{n}_{h}=0.325$ (b), $\bar{n}_{h}=0.525$ (c) and $\bar{n}_{h}=0.725$ (d). All the other parameters are as explained in the text.

where

$$
\begin{gathered}
R=\frac{4}{\pi} \frac{\sqrt{2} \omega_{b} g}{\sqrt{\kappa_{b}^{2}\left(\omega_{b}^{2}+\frac{\kappa_{b}^{2}}{16}\right)}}\left(\frac{\bar{n}_{h}-\bar{n}_{c}}{4}\right), \\
q_{b 0}=\frac{2 \omega_{b} g}{\sqrt{2} \omega^{2}}\left(\frac{\bar{n}_{h}+3 \bar{n}_{c}}{4}\right),
\end{gathered}
$$

and $p_{b 0} \approx 0$.

The effect of coherence in the LF mode state can be further revealed by calculating the zero-time-delay secondorder correlation function $g^{(2)}(0):=\left\langle\hat{b}^{\dagger} \hat{b}^{\dagger} \hat{b} \hat{b}\right\rangle /\left\langle\hat{n}_{b}\right\rangle^{2}$, of the LF mode. Figure 5 shows the time dependence of $g^{(2)}(0)$ of the LF mode for the same $\bar{n}_{h}$ values as in Fig. 2. Initially, $g^{(2)}(0)=2$ for all cases as the LF mode starts in a thermal state. $g^{(2)}(0)$ decrease as the LF mode gains partial coherence in time. At higher $\bar{n}_{h}$, the steady-state value of $g^{(2)}(0)$ gets closer to the coherent state value $g^{(2)}(0)=1$. While perfect coherence is not achieved within the range of values of $\bar{n}_{h}$ considered, a slow convergence to coherent state statistics can be seen in Fig. 5. We note that the second-order coherence function can be measured in circuit QED systems using various techniques such as by linear detectors [60].

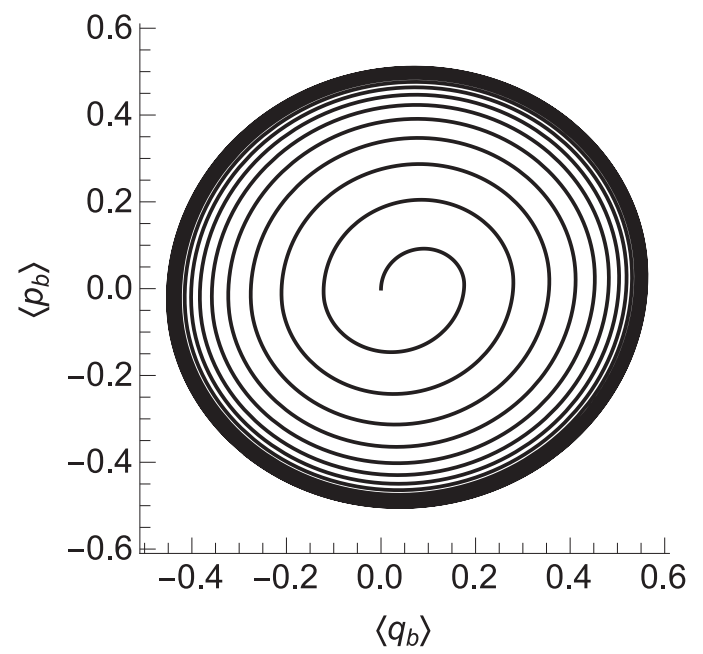

FIG. 4. Phase portrait $\left\langle q_{b}\right\rangle-\left\langle p_{b}\right\rangle$ showing the dynamics of the LF mode for $\bar{n}_{h}=0.125$. All the other parameters are as explained in the text.

\section{B. Effective Otto engine cycle}

In order to describe the engine cycle, we introduce an effective HF mode frequency $\omega_{\text {eff }}:=\omega_{a}-g q$, which can be interpreted as the change in the frequency associated with the variations in the electrical length of the HF resonator. Accordingly, the effective mean energy of the working fluid can be taken to be $U_{a}=\omega_{\text {eff }}\left\langle\hat{n}_{a}\right\rangle$. This factorization ignores the correlations between $\hat{n}_{a}$ and $\hat{q}$. When we calculate $U_{a}$ without the factorization assumption, we find qualitatively the same cycle pictures and the work output is negligibly enhanced. For the moment, we consider the explanation of the engine cycle in this mean-field model where all correlations are neglected and discuss the effect of quantum correlations separately in the later sections.

In Fig. 6, we plot the dependence of $U_{a}$ on $\omega_{\text {eff }}$ in the steady state at $\bar{n}_{h}=0.125$ for the same set of parameters used in earlier figures. We plot $\ln \left(U_{a}\right)$ in order to emphasize that the temperature in the lower branch $\left(\mathrm{D}^{\prime}\right.$ to $\left.\mathrm{A}\right)$ is increasing. A four-stage engine cycle can be identified in this picture.

The first stage is indicated by the arrow from point $\mathrm{A}$ to $\mathrm{B}$ in Fig. 6 at $\omega_{\text {eff }} \sim 1.03$ and corresponds to an isochoric heating of the HF resonator by the incoming heat pulse. The electrical length $1 / \omega_{\text {eff }}$ remains constant while the incoherent energy is received from the external noise pulse. The coherence of the

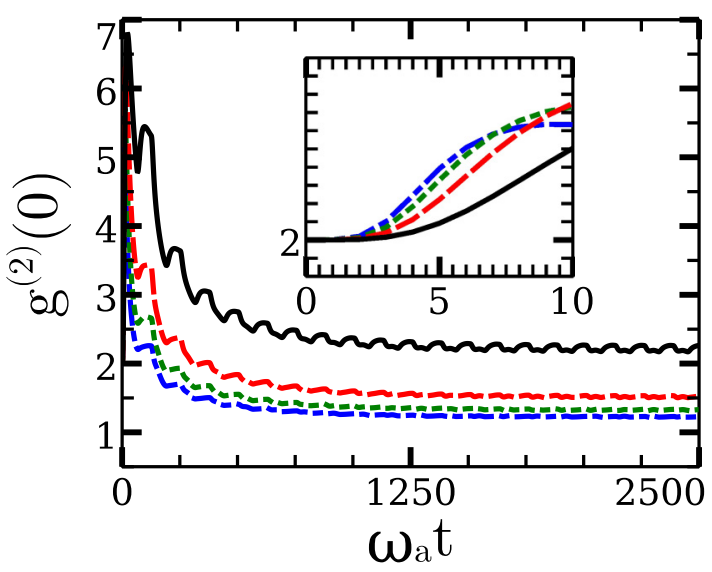

FIG. 5. Time dependence of the zero-time-delay second-order correlation function $g^{(2)}(0)$ of the LF mode for $\bar{n}_{h}=0.125$ (black solid), $\bar{n}_{h}=0.325$ (red dashed), $\bar{n}_{h}=0.525$ (green dotted), and $\bar{n}_{h}=$ 0.725 (blue dot-dashed). The inset is the magnification of $g^{(2)}(0)$ up to $10 \omega_{a} t$. Time is dimensionless and scaled with the HF mode frequency $\omega_{a}$. All the other parameters are as explained in the text. 


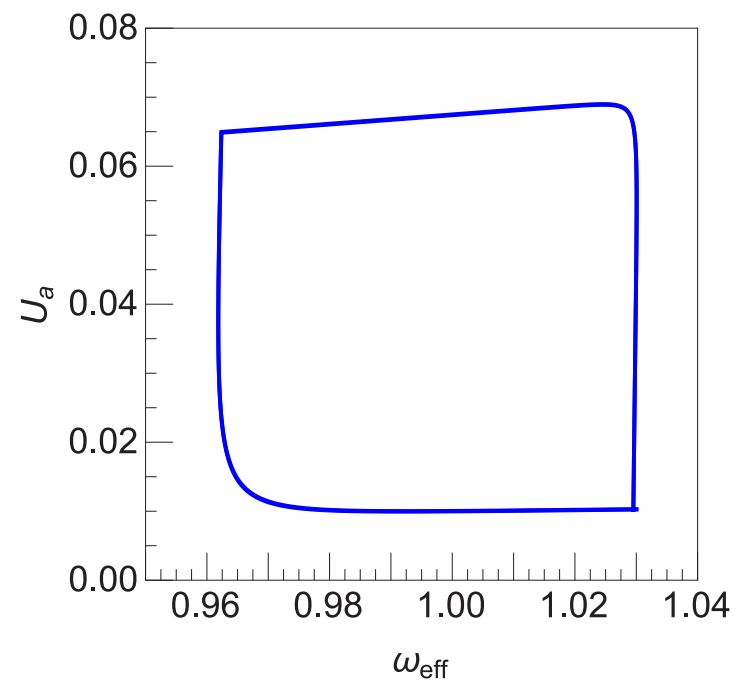

(a)

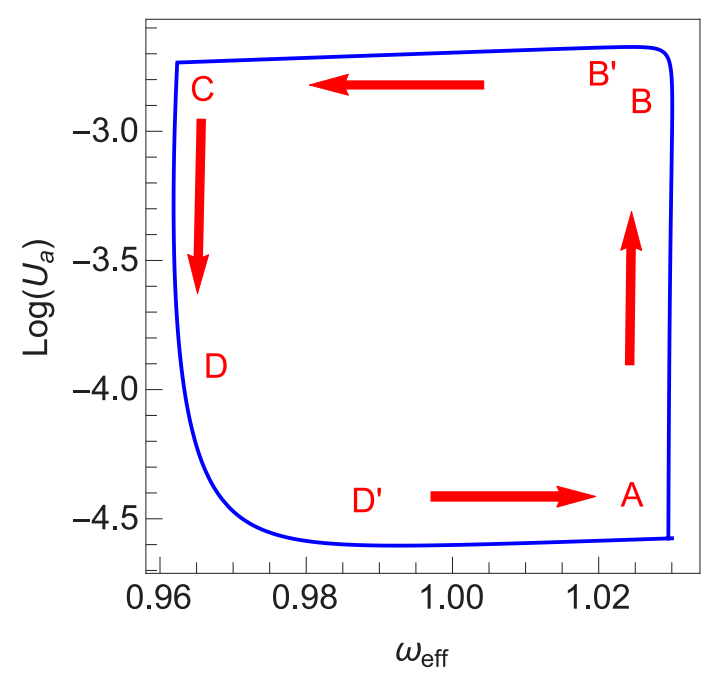

(b)

FIG. 6. (a) Dependence of the effective internal energy of the HF resonator $U_{a}=\omega_{\text {eff }}\left\langle\hat{n}_{a}\right\rangle$ on the effective HF mode frequency $\omega_{\text {eff }}=\omega_{a}-g\left(\langle\hat{b}\rangle+\left\langle\hat{b}^{\dagger}\right\rangle\right)$ for $\bar{n}_{h}=0.125$ in the steady state. (b) The plot shows $\ln \left(U_{a}\right)$ in order to emphasize that the temperature in the lower branch ( $\mathrm{D}^{\prime}$ to $\left.\mathrm{A}\right)$ is increasing. The direction of the engine cycle in the steady state is indicated by the red arrows. The points A, B, $\mathrm{B}^{\prime}, \mathrm{C}, \mathrm{D}$, and $\mathrm{D}^{\prime}$ are used in the text to describe the thermodynamic processes in the cycle. We use the frequency of the HF resonator, $\omega_{a}$, to scale our parameters. All the other parameters are as explained in the text.

LF mode cannot follow the thermalization of the HF mode as fast (cf. Fig. 2) so that the "piston," or the LF mode quadrature, remains at rest in the phase space. There is a transitional stage from $\mathrm{B}$ to $\mathrm{B}^{\prime}$ which cannot be identified with the standard thermodynamical processes.

The second stage, from $\mathrm{B}^{\prime}$ to $\mathrm{C}$, is adiabatic expansion of the $\mathrm{HF}$ resonator, where $\omega_{\text {eff }}$ decreases to $\sim 0.96$, and hence the electrical length of the HF resonator increases. The entropy of the HF resonator field can be calculated by

$$
S_{a}=\left(1+\left\langle\hat{n}_{a}\right\rangle\right) \ln \left(1+\left\langle\hat{n}_{a}\right\rangle\right)-\left\langle\hat{n}_{a}\right\rangle \ln \left(\left\langle\hat{n}_{a}\right\rangle\right),
$$

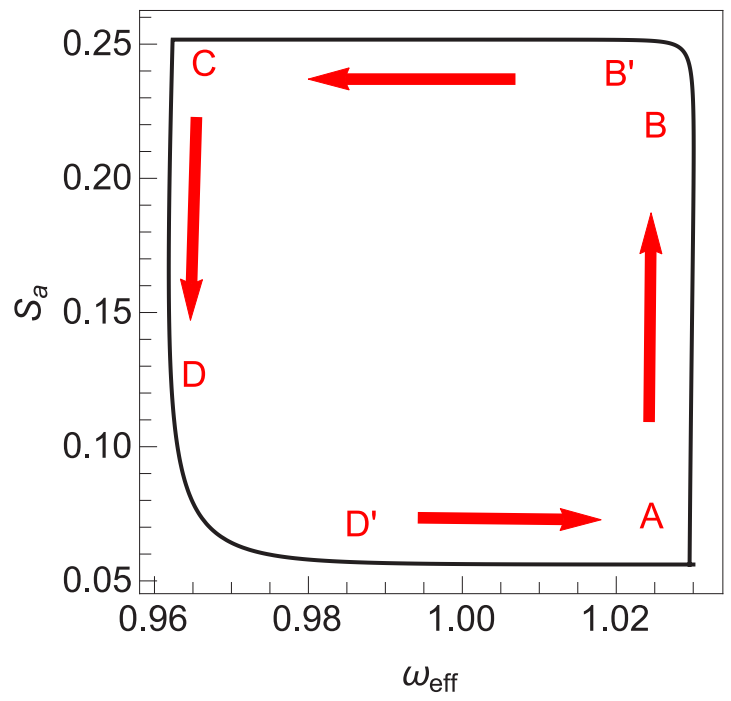

FIG. 7. Steady-state dependence of the entropy $S\left(\rho_{a}\right)$ on the effective HF mode frequency $\omega_{\text {eff }}=\omega_{a}-g\left(\langle\hat{b}\rangle+\left\langle\hat{b}^{\dagger}\right\rangle\right)$ for $\bar{n}_{h}=$ 0.125 . We use the frequency of the HF resonator $\omega_{a}$ to scale our parameters. All the other parameters are as explained in the text.

which remains constant in this stage as shown in Fig. 7. The heating pulse is still active, but the HF mode is in thermal equilibrium and working on the LF mode. The coherence in the LF mode builds up in this stage as the "piston" shifts in the phase space, converting heat to potential energy to be harvested.

The third stage is isochoric cooling that happens from $\mathrm{C}$ to $\mathrm{D}$ at $\omega_{\text {eff }} \sim 0.96$, following the rapid decrease of the population of the HF mode when the heating pulse is turned off (cf. Fig. 2). There is another transitional stage from $\mathrm{D}$ to $\mathrm{D}^{\prime}$. The final stage from $\mathrm{D}^{\prime}$ to A closes the cycle. It corresponds to an adiabatic compression (cf. Fig. 7), where $\omega_{\text {eff }}$ increases to $\sim 1.03$. The sign change in the coherence of the LF mode [cf. Fig. 2(c)] leads to an increasing $\omega_{\text {eff }}$ (decreasing electrical length) so that the "piston" moves back to its original location in the phase space and the cycle is complete.

\section{Performance of the engine}

Our four-stage engine description, other than the transitional stages, can be considered an Otto cycle. The effects of the transitional stages do not strongly influence the temperature-entropy $(T-S)$ diagram plotted in Fig. 8, which closely resembles that of an Otto engine. Here we introduced an effective temperature given by

$$
T_{\mathrm{eff}}=\omega_{\mathrm{eff}} / \ln \left(1+1 /\left\langle\hat{n}_{a}\right\rangle\right) .
$$

The $T$-S diagram in Fig. 8, which follows a narrow cycle, is of a similar form to that obtained experimentally for the single-atom heat engine [40] and for a nanomechanical Otto engine driven by a squeezed reservoir to operate with an efficiency beyond the classical Carnot limit [61]. The area of the effective $T-S$ curve defines the "mechanical output" which is stored in the coherent oscillations of the LF resonator and not dissipated as heat. The cyclic work output of the effective Otto engine periodically drives the coherent oscillations of the LF resonator mode. This does not cause an ever-increasing 


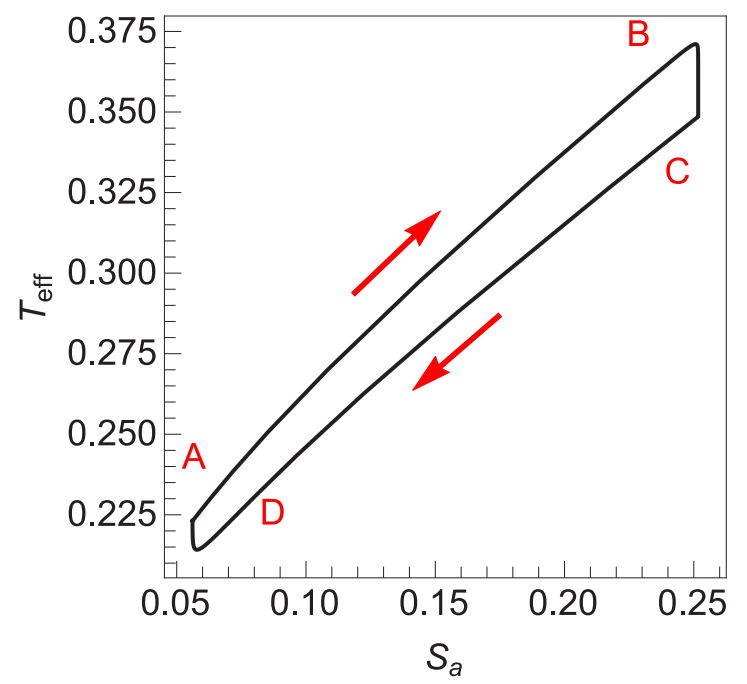

FIG. 8. $T-S$ diagram of the HF mode for $\bar{n}_{h}=0.125$. We use the frequency of the HF resonator, $\omega_{a}$, to scale our parameters. All the other parameters are as explained in the text.

oscillation amplitude as the drive is balanced with the friction effect of the cold baths attached to the LF resonator [cf. Eq. (9)]. The amplitude of the oscillations as well as their coherence can be determined using the standard methods of coherence function measurements in circuit QED [48].

A similar principle of work storage in coherent oscillations is employed in the trapped ion Otto engine experiment [40], where the radial and axial degrees of freedom of the ion are interacting through an optomechanical-like coupling. The radial degree of freedom undergoes closed engine cycles by successive heating and cooling. The work output of the cycles drives the coherent oscillations along the axial direction. The work is stored in the amplitude of the coherent axial oscillations. Since the atomic piston is in principle a frictionless system, the oscillations would increase with time. To avoid this flywheel-like behavior, damping is introduced through an additional cooling laser such that constant amplitude oscillations of the axial mode are achieved in steady state. Similar work storage principles are employed in more recent experiments as well $[62,63]$.

By approximating the $T-S$ diagram in Fig. 8 by a trapezoid, we can estimate the net work by $W_{a} \sim 3.5 \times 10^{-3} \hbar \omega_{a} \sim$ $2.3 \times 10^{-26} \mathrm{~J}$. Dividing by the cycle time, which is the heating pulse period $2 \pi / \omega_{b}=2 \mathrm{~ns}$, we find the power from the working fluid as $P_{a} \sim 1.15 \times 10^{-17} \mathrm{~W}$. The heat intake of the HF resonator can also be determined from the diagram and we find $Q_{\text {in }} \sim 0.06 \hbar \omega_{a}$, which yields an efficiency of $\eta=W_{a} / Q_{\text {in }} \sim 0.06 \%$. Similar values can be consistently found from the cycle in the $\left\langle\hat{n}_{a}\right\rangle-\omega_{\text {eff }}$ diagram, which is similar to Fig. 6. Approximating the cycle by a rectangular path, we can verify that $W_{a} \sim \hbar\left(n_{H}-n_{L}\right)\left(\omega_{H}-\omega_{L}\right)$, where $n_{H} \sim 0.0675$ and $n_{L}=0.01$ (cf. Fig. 2) are the maximum and minimum $\left\langle\hat{n}_{a}\right\rangle$, respectively. Similarly, $\omega_{H} \sim 1.04$ and $\omega_{L} \sim 0.96$ (see, e.g., Fig. 7) are the maximum and minimum of $\omega_{\text {eff }}$, respectively. The heat intake in this case can be written as $Q_{\text {in }} \sim \hbar \omega_{H}\left(n_{H}-n_{L}\right)$. Hence, the efficiency becomes that of an Otto engine $\eta=1-\omega_{L} / \omega_{H} \sim 0.08 \%$. These values increase with $\bar{n}_{h}$. For example, at $\bar{n}_{h}=1$, we get $\eta \sim 0.27 \%, P \sim 2.64 \times 10^{-16} \mathrm{~J} / \mathrm{s}$, and $W \sim 5.28 \times 10^{-25} \mathrm{~J}$. We cannot increase $\bar{n}_{h}$ indefinitely however. Optomechanical model requires relatively small oscillations such that $\omega_{a}>$ $g|q|$ at all times. This limits our considerations to a regime $\bar{n}_{h}<3.25$.

Alternatively, another figure of merit, called the dissipative power, is also considered to estimate the performance of such piston engines, which is given by [27]

$$
\begin{aligned}
P & =-\operatorname{Tr}\left\{\omega_{b} \hat{n}_{b} \kappa_{b}\left[\left(\bar{n}_{b}+1\right) D[\hat{b}]+\bar{n}_{b} D\left[\hat{b}^{\dagger}\right]\right]\right\}, \\
& =\omega_{b} \kappa_{b}\left(\left\langle\hat{n}_{b}\right\rangle-\bar{n}_{c}\right) .
\end{aligned}
$$

It is nonzero when the LF resonator mode is not in equilibrium with the environment. It has the same qualitative behavior with the mean number of excitations in the LF mode by definition and hence is oscillatory around a mean value (cf. Fig. 2). $P$ is approximately the same as the heat current from the LF resonator to its thermal bath, which is given by

$$
J_{b}=\operatorname{Tr}\left[\hat{\mathcal{L}}_{b} \hat{H}_{\mathrm{sys}}\right]=P+g \frac{\kappa_{b}}{2}\left\langle\hat{n}_{a} \hat{q}\right\rangle,
$$

where $\hat{\mathcal{L}}_{b}$ represents all the Lindblad superoperators depending on $\kappa_{b}$. The last term in the $J_{b}$ expression is negligible. The approximation of $J_{b} \approx P$ improves by including the nonlocal effects of the reservoir-system interactions. The last two statements are verified numerically for our parameter regimes using the global master equation given in Appendix A.

We can find a general relation between the mechanical power of the effective Otto engine and the dissipative power. In terms of the electrical pressure $\hat{F} \sim \hat{n}_{a}$ and the electrical length change $d \hat{q}$, we can write a mechanical work operator as $\delta \hat{W}=-g \hat{n}_{a} d \hat{q}$, so that the mechanical power can be expressed as $P_{m}=\langle\delta \hat{W} / \delta t\rangle=-g\left\langle\hat{n}_{a}(d \hat{q} / d t)\right\rangle$. Using $d \hat{q} / d t=\omega_{b} \hat{p}$, mechanical work can be expressed in terms of pressure-momentum correlation as $P_{m}=-g \omega_{b}\left\langle\hat{n}_{a} \hat{p}\right\rangle$. Note that this expression is the same with the variation $d U_{a} / d t$ of the effective mean energy of the HF resonator mode $U_{a}=\omega_{a}\left\langle\hat{n}_{a}\right\rangle-g\left\langle\hat{n}_{a} \hat{q}\right\rangle$ along the quantum adiabats at which $\left\langle\hat{n}_{a}\right\rangle$ is constant. We can now substitute $\left\langle\hat{n}_{b}\right\rangle=P / \omega_{b} \kappa_{b}+\bar{n}_{b}$ into the equation of motion for $\left\langle\hat{n}_{b}\right\rangle$ (see Appendix A), which gives

$$
\frac{d P}{d t}+\kappa_{b} P=-\kappa_{b} P_{m}
$$

If the baths are continously attached to the resonators, without timed sequential heating and cooling stages, then the system operates as a heat transport device and in the steady state we get $P_{m}=-P$. In our case of engine operation, however, $P$ is oscillatory in the steady state [cf. Fig. 2(b)] and the mechanical work is stored in the coherent oscillation amplitude of the LF resonator mode. We can measure $J_{b}$, which is approximately the same as $P$, to assess how large these oscillations are, as they are driving the dissipative power. Note that such a measurement per se would not give information that the stored energy is a coherent and can be considered as work. It should be complemented with a coherence measurement as well (cf. Fig. 5).

Alternatively, the mechanical output could be harvested directly by attaching an electrical load to the open terminal of the LF transmission line resonator [27]. This could change the 


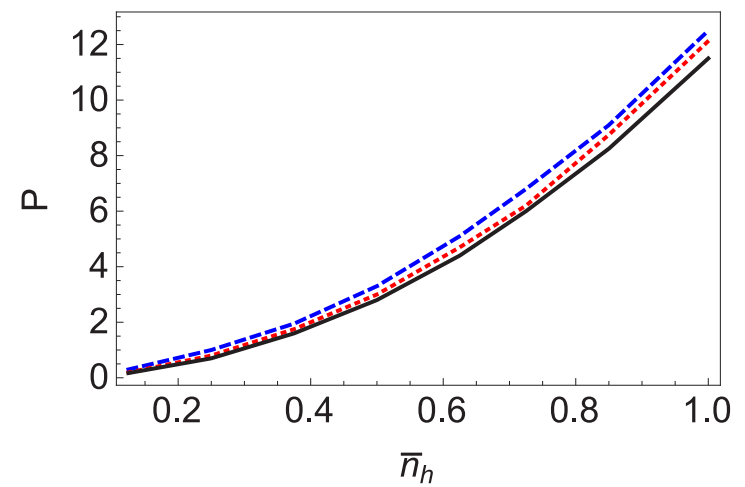

FIG. 9. The maximum power (in units of $\kappa_{b} \hbar \omega_{b}$ ) with respect to average number of excitations $\bar{n}_{h}$ in the effective hot bath. The black solid, red dotted, and blue dashed curves indicate the results of the mean field, classical, and quantum calculations. All the other parameters are as explained in the text.

dynamics we discussed here and would need a separate study. Moreover, there are different definitions of work reservoirs in terms of attached loads and quantum work extraction [27,6466]. Here we will briefly present some intuitive and qualitative discussion. In our case, we can envision a specific scenario to define a task for the "piston resonator mode." For that aim, the power could be extracted from the resonator by attaching a "matched" microwave system. From the point of view of the resonator, this would look like a purely real impedance that matches the characteristic impedance of the transmission line. From basic microwave theory, this is the way to get the maximum power transfer between the two systems. In this way, modeling the system being "powered" as a resistance gives an upper bound on the microwave power we can extract. Still, it is quite a general model, as we can often use resonant impedance transformation techniques to match an arbitrary load.

One more alternative method of work extraction is based on nonpassivity of the state of the LF resonator mode. The mechanical power of the HF resonator is used to "charge" the LF resonator by putting it into a thermal coherent state. Such states belong to the class of so-called thermodynamically nonpassive states and are capable of producing useful work. Disconnecting the LF resonator from the system would allow it to be used as a quantum coherent resource $[27,28]$. In our case we characterize the coherence properties of the LF resonator mode by calculating the second-order coherence, as plotted in Fig. 5. Its measurement can be used to assess the nonpassivity of the LF resonator mode. For this particular scheme of work extraction we would need a tunable version of the coupled resonator system in which the coupling coefficient $g$ can be turned off and the LF resonator would be tuned to couple to another system, which would then be driven coherently $[27,28]$.

We report the maximum power $P_{\max }$, which is evaluated for the maximum $\left\langle n_{b}\right\rangle$ in the steady state, in Fig. 9. After $\bar{n}_{h} \sim 0.325$ the coherent character of the LF mode steady state becomes more significant than its thermal character (cf. Fig. 5), and hence the dissipated power has more useful work content than the incoherent energy. The other two curves in the figure corresponds to the results of the mean field and classical models of the system. The mean-field model,

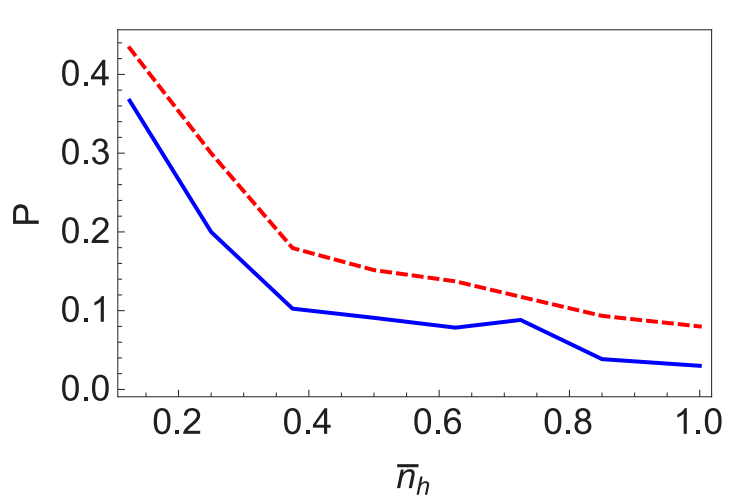

FIG. 10. Behavior of the relative difference in the dissipative power with respect to average number of excitations $\bar{n}_{H}$ in the hot bath. Red dashed and blue solid curves indicate $\left(P_{q}-P_{c}\right) / P_{q}$ and $\left(P_{q}-P_{\mathrm{sc}}\right) / P_{q}$, where $P_{q}, P_{c}$, and $P_{\mathrm{sc}}$ are calculated by quantum, classical, and mean-field methods, respectively. Power is dimensionless and scaled with $\kappa_{b} \hbar \omega_{b}$. All the other parameters are as explained in the text.

as described in the preceding subsection about the engine cycle, ignores the correlations and factorizes the two operator products $\left\langle\hat{n}_{a} q\right\rangle$ and $\left\langle\hat{n}_{a} p\right\rangle$ (see their equations of motion in the Appendix A). We will describe the classical model next and explain the hierarchy of the curves in Fig. 9 in terms of the classical and quantum correlations. We note that as $\bar{n}_{h}$ grows, the quantum correlations are less significant. The relative difference between the classical and quantum descriptions diminish with increasing temperature as shown in Fig. 10.

\section{CLASSICAL MODEL}

In order to distinguish quantum features in our engine system from its classical counterpart, we shall now treat the model Hamiltonian in Eq. (1) as a classical model. Replacing the operators of the fields of the resonators with the $c$ numbers such that $\hat{a} \rightarrow \alpha_{a}$ and $\hat{b} \rightarrow \alpha_{b}$ we get the classical Langevin equations

$$
\begin{aligned}
\dot{\alpha}_{a}= & -\left(i \omega_{a}-i g\left(\alpha_{b}^{*}+\alpha_{b}\right)+\frac{\kappa_{h}}{2}+\frac{\kappa_{a}}{2}\right) \alpha_{a} \\
& +\xi_{h}(t)+\xi_{a}(t), \\
\dot{\alpha}_{b}= & -\left(i \omega_{b}+\frac{\kappa_{b}}{2}\right) \alpha_{b}+i g\left|\alpha_{a}\right|^{2}+\xi_{b}(t),
\end{aligned}
$$

where $\xi_{i}(t)$ with $i=a, b, h$ represents time dependent delta-correlated stochastic noise with $\left\langle\xi_{i}(t)\right\rangle=0$ and $\left\langle\xi_{i}\left(t_{1}\right) \xi_{i}\left(t_{2}\right)\right\rangle:=D_{i} \delta\left(t_{1}-t_{2}\right)$, where $D_{i}$ is the strength of the noise. The parameter $D_{i}$ is a function of $\kappa_{i}$ according to the fluctuation-dissipation theorem [67] such that $D_{i}=\kappa_{i} \bar{n}_{i}$.

We define the field quadratures $X_{i}, Y_{i}$ such that $\alpha_{a}:=$ $1 / \sqrt{2}\left(X_{a}+i Y_{a}\right)$ and $\alpha_{b}:=1 / \sqrt{2}\left(X_{b}+i Y_{b}\right)$. By writing the noise parameters as $\xi_{i}=1 / \sqrt{2}\left(\xi_{x}^{i}+i \xi_{y}^{i}\right)$, we can express the equations to be simulated as

$$
\begin{aligned}
d X_{a} & =\left[\omega_{a} Y_{a}-g \sqrt{2} X_{b} Y_{a}-\kappa_{a}^{\prime} X_{a}\right] d t+d W_{x}^{h}+d W_{x}^{a}, \\
d Y_{a} & =-\left[\omega_{a} X_{a}-g \sqrt{2} X_{a} X_{b}+\kappa_{a}^{\prime} Y_{a}\right] d t+d W_{y}^{h}+d W_{y}^{a},
\end{aligned}
$$




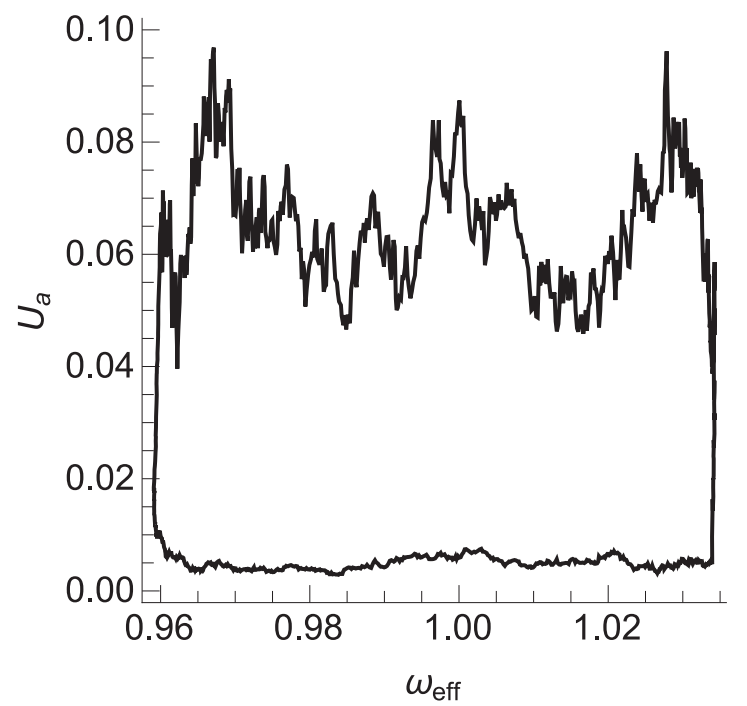

FIG. 11. Change in the internal energy $U$ of the classical engine with respect to the effective frequency $\omega_{\text {eff }}$ for $\bar{n}_{h}=0.125$. We use the frequency of the HF resonator $\omega_{a}$ to make our parameters scaled and dimensionless. All the other parameters are as explained in the text.

$$
\begin{aligned}
& d X_{b}=\left[\omega_{b} Y_{b}-\frac{\kappa_{b}}{2} X_{b}\right] d t+d W_{x}^{b} \\
& d Y_{b}=-\left[\omega_{b} X_{b}+\frac{\kappa_{b}}{2} Y_{b}-\frac{g}{\sqrt{2}}\left(X_{a}^{2}+Y_{a}^{2}\right)\right] d t+d W_{y}^{b} .
\end{aligned}
$$

Here $d W^{i}=1 / \sqrt{2}\left(d W_{x}^{i}+i d W_{y}^{i}\right)$, where $\xi_{k}^{i} d t=: d W_{k}^{i}$, with $k=x, y$, is the Wiener process with width $\sqrt{\kappa_{i} \bar{n}_{i} d t}$ and $\kappa_{a}^{\prime}:=$ $\kappa_{h} / 2+\kappa_{a} / 2$. For the cooling stage, $\kappa_{a}^{\prime}$ is replaced by $\kappa_{a} / 2$ and the $d W_{x}^{h}, d W_{y}^{h}$ terms vanish.

The physical parameters used in the classical dynamical simulations are the same as those used in the quantum case. The equations are solved by using Mathematica 10 .

\section{RESULTS OF CLASSICAL SIMULATIONS}

In Fig. 11, we present the change in the internal energy $U_{a}$ of the classical engine with respect to $\omega_{\text {eff }}$ for $\bar{n}_{h}=0.125$. The result follows and fluctuates about the mean-field model cycle in Fig. 6, as expected theoretically. Similarly, the phase portrait of the classical engine in Fig. 12 is the same with its quantum counterpart in Fig. 4. Theoretically, the quantum dynamics of $\left\langle\hat{n}_{a}\right\rangle$ and the phase-space quadratures are the same for the classical and quantum mechanical descriptions. Factorization of the two operator expectation value $\left\langle\hat{n}_{a} \hat{q}\right\rangle$ to define $\omega_{\text {eff }}$ makes the mean-field model $U_{a}-\omega_{\text {eff }}$ cycle identical with the classical one.

Figure 9 shows the maximum power output of the classical engine with respect to $\bar{n}_{h}$. The power output of the classical engine lies between the quantum and mean-field models. The difference of quantum model from the classical and the mean-field models is more significant in the dissipated power output of the LF resonator in contrast to negligible difference between the classical and quantum treatment of the extractable work from the HF resonator. The differences diminish with increasing temperature of the driving noise as shown in

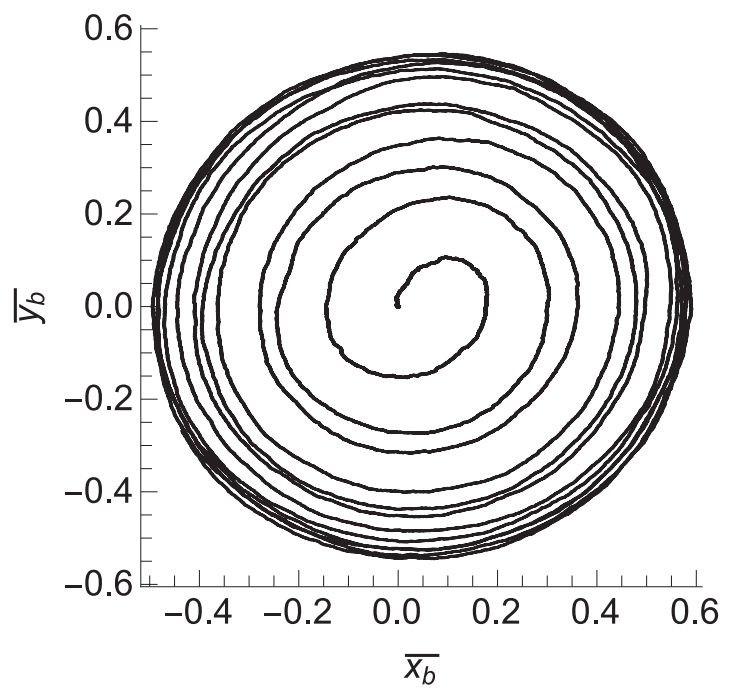

FIG. 12. Phase portrait $X_{b}-Y_{b}$ of the classical LM resonator for $\bar{n}_{h}=0.125$. All the other parameters are as explained in the text.

Fig. 10. In order to explain such different effects of correlations on different subsystems of the engine, we investigate the classical and quantum correlations more closely in the next section. We note that power enhancement in the coherent work extraction relative to the stochastic one has been discussed in the literature as a signature of the quantum character of certain quantum heat engines, different than our system. Quantum coherence has been suggested as the main source of the power enhancement [41]. It has been conjectured that quantum correlations (entanglement or discord) in multiparticle engines could play a similar role to that of coherence in single-particle engines. Our two-coupled-resonator setup can be envisioned as an example to verify this conjecture. As the systems are different, we need to elaborate explicitly if and how the quantum and classical correlations emerge in our system and how they lead to enhanced power output in our case.

\section{QUANTUM NATURE OF THE SYSTEM}

The equations of motion for the dynamical variables of interest in our system are given in Appendix A. In this section, we will discuss the set of equations related to the correlations contributing to the power and work output of our quantum heat engine.

The extractable work depends on the pressure-displacement correlation $\left\langle\hat{n}_{a}, \hat{q}\right\rangle$, where the correlation function for two operators $\hat{o}_{1}$ and $\hat{o}_{2}$ is defined as $\left\langle\hat{o}_{1}, \hat{o}_{2}\right\rangle=\left\langle\hat{o}_{1} \hat{o}_{2}\right\rangle-\left\langle\hat{o}_{1}\right\rangle\left\langle\hat{o}_{2}\right\rangle$. According to Eq. (20), the power output of the Otto cycle of the HF resonator depends on the pressure-momentum correlation $\left\langle\hat{n}_{a}, \hat{p}\right\rangle$. Both the pressure-displacement and pressuremomentum correlations are driven by the number fluctuations of the HF mode which is thermally excited by the hot reservoir. This mechanism is described by the closed system of equations given in Appendix A. We can find the equation of motion for $\left\langle\hat{n}_{a}, \hat{q}\right\rangle$ as

$$
\left(\frac{d^{2}}{d t^{2}}+2 C \frac{d^{2}}{d t}+\omega^{2}\right)\left\langle\hat{n}_{a}, \hat{q}\right\rangle=2 g \omega_{b}\left\langle\left(\Delta \hat{n}_{a}\right)^{2}\right\rangle,
$$




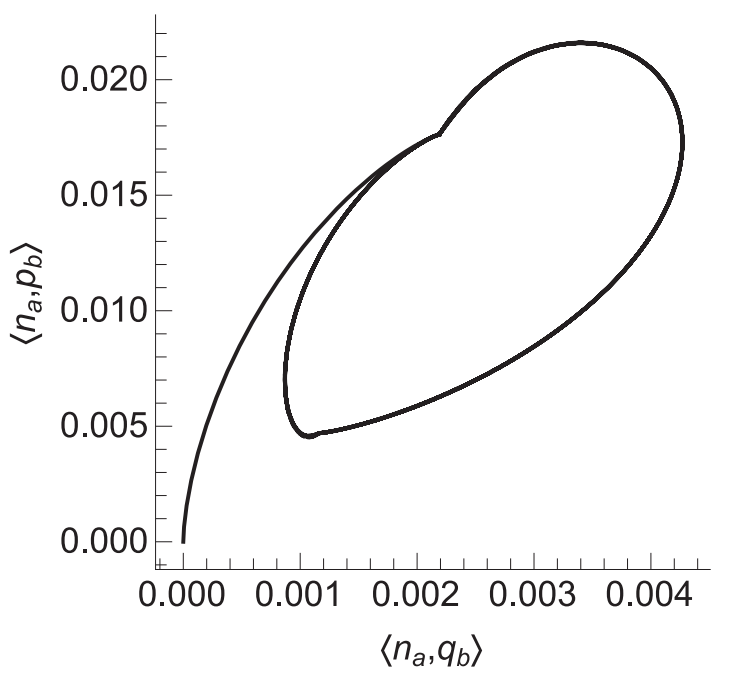

FIG. 13. Relation between the dynamics of the quantum correlations $\left\langle\hat{n}_{a}, \hat{q}\right\rangle$ and $\left\langle\hat{n}_{a}, \hat{q}\right\rangle$. A closed cycle emerges in the steady state as both correlations are slaved by the number fluctuation dynamics of HF resonator. $\left\langle\hat{n}_{a}, \hat{p}\right\rangle$ varies over a much wider range than the $\left\langle\hat{n}_{a}, \hat{q}\right\rangle$. All the other parameters are as explained in the text.

where $C=\kappa_{h}+\kappa_{a}+\kappa_{b} / 2$ and $\omega=\omega_{b}^{2}+C^{2}$. The system of equations for $\left\langle\left(\Delta \hat{n}_{a}\right)^{2}\right\rangle,\left\langle\hat{n}_{a}, \hat{q}\right\rangle$, and $\left\langle\hat{n}_{a}, \hat{p}\right\rangle$ is parallel to that of $\left\langle\hat{n}_{a}\right\rangle,\langle\hat{q}\rangle$, and $\langle\hat{p}\rangle .\left\langle\left(\Delta \hat{n}_{a}\right)^{2}\right\rangle$ and $\left\langle\hat{n}_{a}\right\rangle$ have similar temporal profiles, with the same periodicity but with different amplitudes. Accordingly, $\left\langle\hat{n}_{a}, \hat{q}\right\rangle$ exhibits a periodically driven underdamped oscillatory motion, which is analogous to the dynamics of $q$. In contrast to the case of $q$, however, the weak damping condition $C<\omega$ is weakly satisfied and the correlation dynamics are in a regime which is only slightly beyond critical damping (since $C \sim 2 \kappa_{a} \gg \omega_{b}$ ).

The formal solution of the $\left\langle\hat{n}_{a}, \hat{q}\right\rangle$ can be written as

$$
\left\langle\hat{n}_{a}, \hat{q}\right\rangle=\int_{0}^{t} d t^{\prime} e^{-C\left(t-t^{\prime}\right)} \sin \left[\omega_{b}\left(t-t^{\prime}\right)\right] f\left(t^{\prime}\right),
$$

with $f(t)=2 g\left\langle\left(\Delta \hat{n}_{a}\right)^{2}(t)\right\rangle$. Another difference from the $q$ dynamics, which has a resonant drive, is that the natural frequency $\omega \sim 2 \kappa_{a}$ of the correlation oscillator is far off resonant with the drive frequency $\omega_{b}$. In this case, the fast correlations adiabatically follows the slow fluctuations of the $\mathrm{HF}$ resonator. We can employ integration by parts to the formal solution to show that

$$
\left\langle\hat{n}_{a}, \hat{q}\right\rangle \approx \frac{g \omega_{b}}{2 \kappa_{a}^{2}}\left\langle\left(\Delta \hat{n}_{a}\right)^{2}\right\rangle\left(1-e^{-C t}\right) .
$$

In the long-time limit the steady state at the end of the heating and cooling cycles coincides with the exact steady state for the continuous drive case discussed in Appendix B. In terms of our parameter values, this value is too small $\left(\sim 10^{-3}\right)$ to be of significance for the extractable work from the working HF resonator. On the other hand, using the relation

$$
\left\langle\hat{n}_{a}, \hat{p}\right\rangle=\frac{1}{\omega_{b}}\left(\frac{d}{d t}+C\right)\left\langle\hat{n}_{a}, \hat{q}\right\rangle
$$

we get

$$
\left\langle\hat{n}_{a}, \hat{p}\right\rangle=\frac{g}{\kappa_{a}}\left\langle\left(\Delta \hat{n}_{a}\right)^{2}\right\rangle,
$$

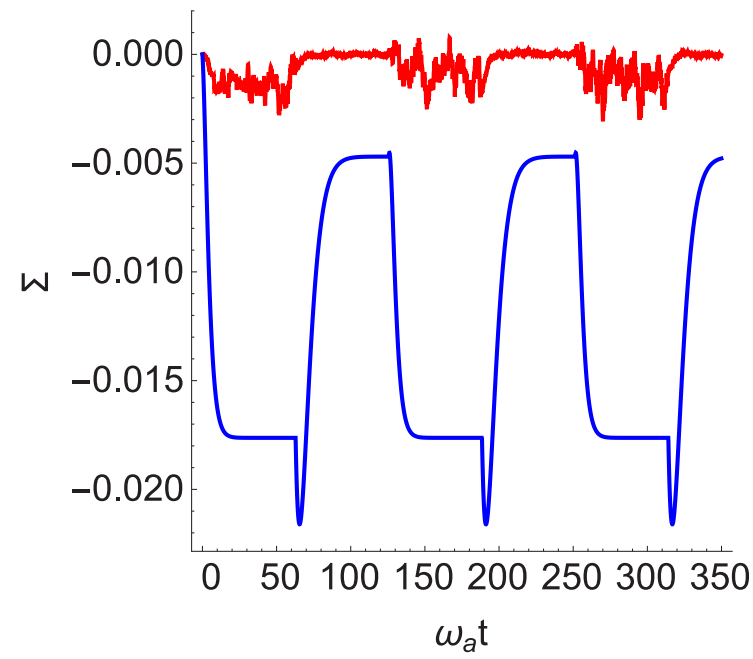

(a)

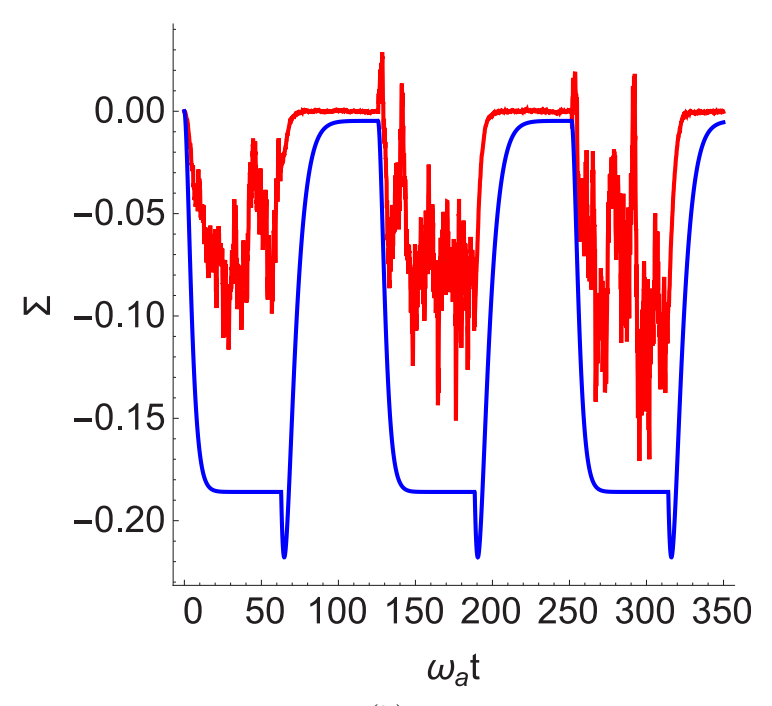

(b)

FIG. 14. Dynamics of the correlation function $\Sigma$ with respect to the scaled time $\omega_{a} t$ for (a) $\bar{n}_{h}=0.125$ and (b) $\bar{n}_{h}=1$ calculated by quantum (solid blue curves) and classical (red noisy curves) methods. All the other parameters are as explained in the text.

which turns out to be an order of magnitude greater than $\left\langle\hat{n}_{a}, \hat{q}\right\rangle$. Their mutual dynamics are plotted in Fig. 13. According to Eq. (20), instead of contributing to the work output, $\left\langle\hat{n}_{a}, \hat{p}\right\rangle$ contributes to power output of the Otto cycle of the HF mode.

We note that a similar parameter to $\left\langle\hat{n}_{a}, \hat{p}\right\rangle$ (normalized by the variances of the $\hat{n}_{a}$ and $\hat{p}$ ) has been proposed in the context of the quality of quantum nondemolition measurements and called the signal-meter entanglement parameter [46]. To make an analogous notation we use $\Sigma:=-\left\langle\hat{n}_{a}, \hat{p}\right\rangle$. This has also been used in optomechanical systems as an indicator of quantum coherence [47]. When $\Sigma<0$, signal-meter type quantum correlations make a positive contribution to the rate of increase of the power output from the LF resonator. The time dependence of $\Sigma$ is shown in in Fig. 14. It is oscillatory, adiabatically following the number fluctuations in the HF resonator, and it is always negative. Similar oscillations in $\Sigma$ are found for a typical optomechanical setup where the drive 
is coherent. It was proposed that the correlated state can be used to generate a mixture of Cat states through conditional measurement [47]. In our case, coherence and correlations are driven by an incoherent drive.

We have also calculated the classical correlation $\Sigma_{\text {class }}=$ $-\left\langle\hat{n}_{a}, \hat{p}\right\rangle_{\text {class. }}$. Here $\langle.\rangle_{\text {class }}$ indicates averaging over classical trajectories. The results are plotted in Fig. 14. This shows that there is a difference between the amplitudes of the quantum and classical correlations. The quantum model predicts a faster growth rate and higher maximum value for the power output from the LF resonator relative to the classical model. Both models predict more powerful and faster engines relative to the mean-field model.

\section{CONCLUSIONS}

In summary, we proposed and investigated a pair of superconducting transmission line resonators with an optomechanical-like interaction as a quantum heat engine. The HF resonator is periodically coupled to an effective hot bath while both resonators are coupled to continuous effective cold baths. We found the emergence of a limit cycle in the open system dynamics and identified that the HF resonator mode undergoes an effective Otto cycle. The electromagnetic field mode of the HF resonator acts as the working substance while the mode of the LF one acts as the piston that can be used for coherent power extraction. The superconducting resonator engine serves as an electrical analog of a mechanical piston engine. We found that a pistonlike motion can be identified in the phase space by using the phase portraits and Wigner function plots. We numerically verified, by calculating the second-order coherence function, that the working resonator remains thermal while the "piston" resonator is "charged" to a thermal coherent state, which is a thermodynamically nonpassive state that can be used to harvest useful work [28].

Extractable work and efficiency has been calculated from the $T-S$ diagram and from the internal energy diagram. We found that pressure-displacement correlations could in principle contribute to the enhancement of extractable work. However, the effect is negligible. A practical figure of merit for the piston engine performance is the dissipative power from the piston subsystem. When we evaluate it, we found that pressure-momentum correlations (also known as signal-meter type correlations) contribute significantly. These correlations are driven by the variance of the working resonator, which is driven by the thermal fluctuations of the hot reservoir. By this way, in addition to the mean number of excitations in the hot bath, its fluctuations can be harvested through the dissipative power of the piston resonator. This is not a universal conclusion. In other optomechanical quantum piston engines quantum correlations could lead to a power increase only in limited parameter regimes [27] or could even be harmful to the output power [35]. We compared the classical, mean-field, and quantum engine descriptions and concluded that our engine is inevitably a genuine quantum heat engine with enhanced power output due to quantum correlations so that it could be used as a test bed to explore quantum effects in quantum engine cycles.

Here we have only considered classical noise drives; however, our framework is applicable to the case of quantum noise drives as well. According to our analytical results, we conclude that the quantum enhancement in the power output can be further increased by using quantum drives instead of classical drives. For example, using a squeezed thermal noise, number fluctuations could be increased to enlarge the pressure-displacement or pressure-momentum correlations to make the quantum enhancement in the work or power output more significant relative to classical engines [29,61,68].

Our results could be practically significant for realization of compact, on-chip, scalable, electronic realizations of genuine quantum heat engines with quantum coherence and correlations advantages relative to their classical counterparts. It can also fundamentally be used as a testbed to explore the quantum-to-classical transition of heat engines and to illuminate the role of quantum correlations in the operation of quantum heat engines.

\section{ACKNOWLEDGMENTS}

We acknowledge Pol Forn-Diaz, Kay Brandner, and Mika Sillanpää for useful discussions. A.Ü.C.H. acknowledges support from the Villum Foundation through a postdoctoral block stipend. Ö.E.M. and A.Ü.C.H. acknowledge the support by the University Research Agreement between Lockheed Martin Chief Scientist's Office and Koç University. C.M.W. acknowledges the support by the University Research Agreement between Lockheed Martin Chief Scientist's Office and University of Waterloo. We acknowledge an anonymous referee for recommending to us Refs. [54,58].

\section{APPENDIX A: QUANTUM DYNAMICS OF THE MODEL SYSTEM}

A "gobal" master equation, also called the "dressed state master equation" (DSME), which is applicable to a system with arbitrarily strong optomechanical coupling, has been derived in Ref. [55] and expressed in the Schroödinger picture as $\left(\hbar=1, k_{B}=1\right)$

$$
\begin{aligned}
\dot{\hat{\rho}}= & -i\left[\hat{H}_{\mathrm{sys}}, \hat{\rho}\right]+\kappa_{a}\left(\bar{n}_{a}+1\right) D[\hat{a}]+\kappa_{a} \bar{n}_{a} D\left[\hat{a}^{\dagger}\right] \\
& +\kappa_{h}(t)\left(\bar{n}_{h}+1\right) D[\hat{a}]+\kappa_{h}(t) \bar{n}_{h} D\left[\hat{a}^{\dagger}\right]+\alpha^{2} \kappa_{d} D\left[\hat{a}^{\dagger} \hat{a}\right] \\
& +\kappa_{b}\left(\bar{n}_{b}+1\right) D\left[\hat{b}-\alpha \hat{a}^{\dagger} \hat{a}\right]+\kappa_{b} \bar{n}_{b} D\left[\hat{b}^{\dagger}-\alpha \hat{a}^{\dagger} \hat{a}\right],
\end{aligned}
$$

where $\alpha=g / \omega_{b}$ characterizes the "nonlocal" effects of the reservoirs and $\kappa_{d}=4 \kappa_{b} T_{b} / \omega_{b}$ is the dephasing rate of the HF resonator mode. This expression is obtained under the assumption that the bath of the LF resonator has an Ohmic spectral density. The interaction of the HF resonator mode with the baths attached to it remain approximately local as $\omega_{a} \gg \omega_{b}$ so that "phonon" side modes do not change the spectral densities of the baths of the HF resonator. This requires the spectral densities to be slowly varying near the resonance frequency $\omega_{a}$. These are consistent with the conditions we employ on the noise drives applied to the resonators.

Equations of motions for the relevant dynamical observables of our system are determined from the master equation 
and given by

$$
\begin{aligned}
\frac{d}{d t}\left\langle\hat{n}_{a}\right\rangle & =A-B\left\langle\hat{n}_{a}\right\rangle, \\
\frac{d}{d t}\langle\hat{p}\rangle= & -\omega_{b}\langle\hat{q}\rangle-\frac{\kappa_{b}}{2}\langle\hat{p}\rangle+2 g\left\langle\hat{n}_{a}\right\rangle, \\
\frac{d}{d t}\langle\hat{q}\rangle= & \omega_{b}\langle\hat{p}\rangle-\frac{\kappa_{b}}{2}\langle\hat{q}\rangle+\alpha \kappa_{b}\left\langle\hat{n}_{a}\right\rangle, \\
\frac{d}{d t}\left\langle\left(\Delta \hat{n}_{a}\right)^{2}\right\rangle= & A+(2 A+B)\left\langle\hat{n}_{a}\right\rangle-2 B\left\langle\left(\Delta \hat{n}_{a}\right)^{2}\right\rangle, \\
\frac{d}{d t}\left\langle\hat{n}_{a}, \hat{p}\right\rangle= & -C\left\langle\hat{n}_{a}, \hat{p}\right\rangle-\omega_{b}\left\langle\hat{n}_{a}, \hat{q}\right\rangle+2 g\left\langle\left(\Delta \hat{n}_{a}\right)^{2}\right\rangle, \\
\frac{d}{d t}\left\langle\hat{n}_{a}, \hat{q}\right\rangle= & -C\left\langle\hat{n}_{a}, \hat{q}\right\rangle+\omega_{b}\left\langle\hat{n}_{a}, \hat{p}\right\rangle+\alpha \kappa_{b}\left\langle\left(\Delta \hat{n}_{a}\right)^{2}\right\rangle, \\
\frac{d}{d t}\left\langle\hat{n}_{b}\right\rangle= & -\kappa_{b}\left(\left\langle\hat{n}_{b}\right\rangle-\bar{n}_{b}\right)+g\left(\left\langle\hat{n}_{a}, \hat{p}\right\rangle+\left\langle\hat{n}_{a}\right\rangle\langle\hat{p}\rangle\right) \\
& +\alpha \frac{\kappa_{b}}{2}\left(\left\langle\hat{n}_{a}, \hat{q}\right\rangle+\left\langle\hat{n}_{a}\right\rangle\langle\hat{q}\rangle\right),
\end{aligned}
$$

where $\hat{q}=\hat{b}+\hat{b}^{\dagger}, \hat{p}=i\left(\hat{b}-\hat{b}^{\dagger}\right), \quad A=\kappa_{a} \bar{n}_{a}+\kappa_{h} \bar{n}_{h}, B=$ $\kappa_{a}+\kappa_{h}$, and $C=B+\kappa_{b} / 2$. Correlation function between the two dynamical observables with the operators $\hat{o}_{1}$ and $\hat{o}_{2}$ are denoted by $\left\langle\hat{o}_{1}, \hat{o}_{2}\right\rangle=\left\langle\hat{o}_{1} \hat{o}_{2}\right\rangle-\left\langle\hat{o}_{1}\right\rangle\left\langle\hat{o}_{2}\right\rangle$. The terms with parameter $\alpha$ are due to the nonlocal effects of the reservoir-system interactions. In the range of our interest, $g \leqslant \omega_{b}$, we did not find any significant effects resulting from them, except a negligible increase in $\left\langle\hat{n}_{a}, \hat{q}\right\rangle$. We use the local master equation and the corresponding equations of motion by taking $\alpha=0$ unless otherwise noted in the paper.

We note that the closed dynamics that we have found for the set of thermodynamically relevant observables cannot be found for all dynamical variables of the system. The optomechanical Hamiltonian has a special symmetry in which the number operator of the HF resonator mode is a constant of motion. The steady state of the $\mathrm{HF}$ resonator and the associated local observables for the HF mode are independent of the optomechanical coupling coefficient $g$ [69]. The first three equations of motion form a closed set of dynamics for the $q-p$ phase-space trajectories, driven by $\left\langle\hat{n}_{a}\right\rangle$. The second set of three equations form another, independent, closed set of dynamics for the "pressure-displacement," $\left\langle\hat{n}_{a}, \hat{q}\right\rangle$, and "pressuremomentum," $\left\langle\hat{n}_{a}, \hat{p}\right\rangle$ correlations, driven by the quantum fluctuations $\left\langle\left(\Delta \hat{n}_{a}\right)^{2}\right\rangle$. The baths of the HF resonator mode prepare the pressure $\left(\sim\left\langle\hat{n}_{a}\right\rangle\right.$ and quantum noise drives for these independent dynamics. Both dynamics contribute to the evolution of the excitations in the LF mode given in the last equation.

\section{APPENDIX B: STEADY STATE OF THE QUANTUM DYNAMICS UNDER CONTINUOUS HEATING}

While the dynamics of the LF mode is different under pulsed thermal drive, the HF resonator reaches approximately its steady state identical with a continuous drive case, for the pulse duration is longer than its thermalization transition time. In order to highlight the effect of pulsed drive relative to continuous drive on the LF mode dynamics, and to give steady-state values of the HF dynamics as well, we provide the solutions of the quantum dynamical equations in Appendix $\mathrm{A}$ in the long-time limit below and note some common characteristic behaviors,

$$
\begin{aligned}
\left\langle\hat{n}_{a}\right\rangle_{\mathrm{SS}}= & \frac{A}{B}=\frac{\kappa_{a} \bar{n}_{a}+\kappa_{h} \bar{n}_{h}}{\kappa_{a}+\kappa_{h}}, \\
\langle\hat{q}\rangle_{\mathrm{SS}}= & \frac{8 g \omega_{b}+4 \alpha \kappa_{b}^{2}}{4 \omega_{b}^{2}+\kappa_{b}^{2}}\left\langle\hat{n}_{a}\right\rangle_{\mathrm{SS}}, \\
\langle\hat{p}\rangle_{\mathrm{SS}}= & \frac{4 g \kappa_{b}-4 \alpha \kappa_{b} \omega_{b}}{4 \omega_{b}^{2}+\kappa_{b}^{2}}\left\langle\hat{n}_{a}\right\rangle_{\mathrm{SS}}=0, \\
\left\langle\left(\Delta \hat{n}_{a}\right)^{2}\right\rangle_{\mathrm{SS}}= & \frac{A(A+B)}{B^{2}}, \\
\left\langle\hat{n}_{a}, \hat{p}\right\rangle_{\mathrm{SS}}= & \frac{2 g C-\alpha \kappa_{b} \omega_{b}}{\omega_{b}^{2}+C^{2}}\left\langle\left(\Delta \hat{n}_{a}\right)^{2}\right\rangle_{\mathrm{SS}}, \\
\left\langle\hat{n}_{a}, \hat{q}\right\rangle_{\mathrm{SS}}= & \frac{2 g B}{\omega_{b}^{2}+C^{2}}\left\langle\left(\Delta \hat{n}_{a}\right)^{2}\right\rangle_{\mathrm{SS}}, \\
\left\langle\hat{n}_{b}\right\rangle_{\mathrm{SS}}= & \left.\left.\bar{n}_{b}+\frac{g}{\omega_{b}^{2}+\left(\left\langle\hat{n}_{a}, \hat{p}\right\rangle_{\mathrm{SS}}+\left\langle\kappa_{b} C\right.\right.}\right\rangle_{\mathrm{SS}}\langle\hat{p}\rangle_{\mathrm{SS}}\right) \\
& +\frac{\alpha}{2}\left(\left\langle\hat{n}_{a}, \hat{q}\right\rangle+\left\langle\hat{n}_{a}\right)^{2}\right\rangle_{\mathrm{SS}},
\end{aligned}
$$

According to our parameters we have $\kappa_{a}=\kappa_{h}$ so that $\left\langle\hat{n}_{a}\right\rangle_{\mathrm{SS}}=$ $\left(\bar{n}_{c}+\bar{n}_{h}\right) / 2$. In addition, the variance becomes $\left\langle\left(\Delta \hat{n}_{a}\right)^{2}\right\rangle_{\mathrm{SS}} \sim$ $\left(\bar{n}_{a}+\bar{n}_{h}\right)\left(\bar{n}_{a}+\bar{n}_{h}+2\right) / 4$. Exact solution of the pulsed-drive case yields these value to a very good approximation, conforming that the pulse duration is sufficiently long to thermalize the $\mathrm{HF}$ resonator. The steady-state values of the LF resonator gives smaller $\left\langle\hat{n}_{b}\right\rangle_{\mathrm{SS}}$ in the case of continuous drive. A common behavior under both the continuous or pulsed drive is the quadratic increase of $\left\langle\hat{n}_{b}\right\rangle_{\text {ss }}$ with $\bar{n}_{h}$. Both the correlation term $\left\langle\hat{n}_{a}, \hat{p}\right\rangle_{\mathrm{SS}}$ and the factorized (mean-field) term $\left\langle\hat{n}_{a}\right\rangle_{\mathrm{SS}}\langle\hat{p}\rangle_{\mathrm{SS}}$ increase quadratically with $\bar{n}_{h}$. This behavior is translated to the power output of the engine, which is proportional to $P \propto\left\langle\hat{n}_{b}\right\rangle_{\mathrm{SS}}-\bar{n}_{b}$.

\section{APPENDIX C: STEADY STATE OF THE ELECTRIC DISPLACEMENT UNDER PERIODIC HEATING}

The equation motion of $q$,

$$
\ddot{q}+\kappa_{b} \dot{q}+\omega^{2} q=2 \omega_{b} g\left\langle n_{a}(t)\right\rangle,
$$

with $\omega^{2}=\omega_{b}^{2}+\kappa_{b}^{2} / 4$, can be solved in steady state by approximating the $\left\langle\hat{n}_{a}\right\rangle$ by a square wave with period $T=$ $2 \pi / \omega_{b}$. Transforming $q \rightarrow q=Q+q_{0}$, we write

$$
\ddot{Q}+\kappa_{b} \dot{Q}+\omega^{2} Q=2 \omega_{b} g\left\langle F_{a}(t)\right\rangle,
$$

where $F(t)$ is made to be periodically alternating between $\pm\left(\bar{n}_{h}-\bar{n}_{c}\right) / 4$ by taking

$$
q_{0}=\frac{2 \omega_{b} g}{\omega^{2}}\left(\frac{\bar{n}_{h}+3 \bar{n}_{c}}{4}\right) .
$$

First harmonic at frequency $\omega_{b}$ is then given by

$$
Q=\frac{4}{\pi} \frac{2 \omega_{b} g}{\sqrt{\kappa_{b}^{2}\left(\omega_{b}^{2}+\frac{\kappa_{b}^{2}}{16}\right)}}\left(\frac{\bar{n}_{h}-\bar{n}_{c}}{4}\right) \sin \left(\omega_{b} t+\alpha\right),
$$

where

$$
\alpha=\arctan \left(\frac{\kappa_{b} \omega_{b}}{\omega_{b}^{2}-\omega^{2}}\right) .
$$


[1] T. D. Kieu, The Second Law, Maxwell's Demon, and Work Derivable from Quantum Heat Engines, Phys. Rev. Lett. 93, 140403 (2004).

[2] H. T. Quan, Y.-x. Liu, C. P. Sun, and F. Nori, Quantum thermodynamic cycles and quantum heat engines, Phys. Rev. E 76, 031105 (2007).

[3] H. T. Quan, P. Zhang, and C. P. Sun, Quantum heat engine with multilevel quantum systems, Phys. Rev. E 72, 056110 (2005).

[4] O. Abah, J. Roßnagel, G. Jacob, S. Deffner, F. Schmidt-Kaler, K. Singer, and E. Lutz, Single-Ion Heat Engine at Maximum Power, Phys. Rev. Lett. 109, 203006 (2012).

[5] C. Bergenfeldt, P. Samuelsson, B. Sothmann, C. Flindt, and M. Büttiker, Hybrid Microwave-Cavity Heat Engine, Phys. Rev. Lett. 112, 076803 (2014).

[6] M. J. Henrich, G. Mahler, and M. Michel, Driven spin systems as quantum thermodynamic machines: Fundamental limits, Phys. Rev. E 75, 051118 (2007).

[7] R. Uzdin and R. Kosloff, The multilevel four-stroke swap engine and its environment, New J. Phys. 16, 095003 (2014).

[8] M. O. Scully, M. S. Zubairy, G. S. Agarwal, and H. Walther, Extracting work from a single heat bath via vanishing quantum coherence, Science 299, 862 (2003).

[9] M. O. Scully, K. R. Chapin, K. E. Dorfman, M. B. Kim, and A. Svidzinsky, Quantum heat engine power can be increased by noise-induced coherence, Proc. Natl. Acad. Sci. USA 108, 15097 (2011).

[10] M. O. Scully, Quantum Photocell: Using Quantum Coherence to Reduce Radiative Recombination and Increase Efficiency, Phys. Rev. Lett. 104, 207701 (2010).

[11] N. Brunner, N. Linden, S. Popescu, and P. Skrzypczyk, Virtual qubits, virtual temperatures, and the foundations of thermodynamics, Phys. Rev. E 85, 051117 (2012).

[12] G. Manzano, F. Galve, R. Zambrini, and J. M. R. Parrondo, Entropy production and thermodynamic power of the squeezed thermal reservoir, Phys. Rev. E 93, 052120 (2016).

[13] Q. Song, S. Singh, K. Zhang, W. Zhang, and P. Meystre, One qubit and one photon-The simplest polaritonic heat engine, Phys. Rev. A 94, 063852 (2017).

[14] F. Altintas, A. Ü. C. Hardal, and Ö. E. Müstecaplıoğlu, Rabi model as a quantum coherent heat engine: From quantum biology to superconducting circuits, Phys. Rev. A 91, 023816 (2015).

[15] E. A. Ivanchenko, Quantum Otto cycle efficiency on coupled qudits, Phys. Rev. E 92, 032124 (2015).

[16] F. Altintas, A. Ü. C. Hardal, and Ö. E. Müstecaplıglu, Quantum correlated heat engine with spin squeezing, Phys. Rev. E 90, 032102 (2014).

[17] C. B. Dağ, W. Niedenzu, Ö. E. Müstecaploğlu, and G. Kurizki, Multiatom quantum coherences in micromasers as fuel for thermal and nonthermal machines, Entropy 18, 244 (2016).

[18] D. Türkpençe and Ö. E. Müstecaplıŏglu, Quantum fuel with multilevel atomic coherence for ultrahigh specific work in a photonic carnot engine, Phys. Rev. E 93, 012145 (2016).

[19] A. Ü. C. Hardal and Ö. E. Müstecaplığlu, Superradiant quantum heat engine, Sci. Rep. 5, 12953 (2015).

[20] M. Campisi and R. Fazio, The power of a critical heat engine, Nat. Commun. 7, 11895 (2016).

[21] R. Kosloff and A. Levy, Quantum heat engines and refrigerators: Continuous devices, Annu. Rev. Phys. Chem. 65, 365 (2014).
[22] F. Tonner and G. Mahler, Autonomous quantum thermodynamic machines, Phys. Rev. E 72, 066118 (2005).

[23] J. Anders and V. Giovannetti, Thermodynamics of discrete quantum processes, New J. Phys. 15, 033022 (2013).

[24] K. Zhang, F. Bariani, and P. Meystre, Quantum Optomechanical Heat Engine, Phys. Rev. Lett. 112, 150602 (2014).

[25] K. Zhang, F. Bariani, and P. Meystre, Theory of an optomechanical quantum heat engine, Phys. Rev. A 90, 023819 (2014).

[26] K. Zhang and W. Zhang, Quantum optomechanical straight-twin engine, Phys. Rev. A 95, 053870 (2017).

[27] A. Mari, A. Farace, and V. Giovannetti, Quantum optomechanical piston engines powered by heat, J. Phys. B 48, 175501 (2015).

[28] D. Gelbwaser-Klimovsky and G. Kurizki, Work extraction from heat-powered quantized optomechanical setups, Sci. Rep. 5, 7809 (2015).

[29] J. Roßnagel, O. Abah, F. Schmidt-Kaler, K. Singer, and E. Lutz, Nanoscale Heat Engine Beyond the Carnot Limit, Phys. Rev. Lett. 112, 030602 (2014).

[30] A. E. Allahverdyan and T. M. Nieuwenhuizen, Extraction of Work From a Single Thermal Bath in the Quantum Regime, Phys. Rev. Lett. 85, 1799 (2000).

[31] K. Korzekwa, M. Lostaglio, J. Oppenheim, and D. Jennings, The extraction of work from quantum coherence, New J. Phys. 18, 023045 (2016).

[32] M. Perarnau-Llobet, K. V. Hovhannisyan, M. Huber, P. Skrzypczyk, N. Brunner, and A. Acín, Extractable Work from Correlations, Phys. Rev. X 5, 041011 (2015).

[33] F. Plastina, A. Alecce, T. J. G. Apollaro, G. Falcone, G. Francica, F. Galve, N. Lo Gullo, and R. Zambrini, Irreversible Work and Inner Friction in Quantum Thermodynamic Processes, Phys. Rev. Lett. 113, 260601 (2014).

[34] A. d. Campo, J. Goold, and M. Paternostro, More bang for your buck: Super-adiabatic quantum engines, Sci. Rep. 4, 6208 (2014).

[35] A. Roulet, S. Nimmrichter, J. M. Arrazola, S. Seah, and V. Scarani, Autonomous rotor heat engine, Phys. Rev. E 95, 062131 (2017).

[36] P. P. Hofer, J.-R. Souquet, and A. A. Clerk, Quantum heat engine based on photon-assisted cooper pair tunneling, Phys. Rev. B 93, 041418 (2016).

[37] P. P. Hofer, J. B. Brask, M. Perarnau-Llobet, and N. Brunner, Quantum Thermal Machine as a Thermometer, Phys. Rev. Lett. 119, 090603 (2017).

[38] P. P. Hofer, M. Perarnau-Llobet, J. B. Brask, R. Silva, M. Huber, and N. Brunner, Autonomous quantum refrigerator in a circuit qed architecture based on a Josephson junction, Phys. Rev. B 94, 235420 (2016).

[39] B. Karimi and J. P. Pekola, Otto refrigerator based on a superconducting qubit: Classical and quantum performance, Phys. Rev. B 94, 184503 (2016).

[40] J. Roßnagel, S. T. Dawkins, K. N. Tolazzi, O. Abah, E. Lutz, F. Schmidt-Kaler, and K. Singer, A single-atom heat engine, Science 352, 325 (2016).

[41] R. Uzdin, A. Levy, and R. Kosloff, Equivalence of Quantum Heat Machines, and Quantum-Thermodynamic Signatures, Phys. Rev. X 5, 031044 (2015).

[42] V. Mukherjee, W. Niedenzu, A. G. Kofman, and G. Kurizki, Speed and efficiency limits of multilevel incoherent heat engines, Phys. Rev. E 94, 062109 (2016). 
[43] H. T. Quan, P.Zhang, and C. P. Sun, Quantum-classical transition of photon-Carnot engine induced by quantum decoherence, Phys. Rev. E 73, 036122 (2006).

[44] J. R. Johansson, G. Johansson, and F. Nori, Optomechanical-like coupling between superconducting resonators, Phys. Rev. A 90, 053833 (2014).

[45] G. Francica, J. Goold, and F. Plastina, The role of coherence in the non-equilibrium thermodynamics of quantum systems, arXiv:1707.06950 (2017).

[46] M. J. Holland, M. J. Collett, D. F. Walls, and M. D. Levenson, Nonideal quantum nondemolition measurements, Phys. Rev. A 42, 2995 (1990).

[47] S. Mancini, V. I. Man'ko, and P. Tombesi, Ponderomotive control of quantum macroscopic coherence, Phys. Rev. A 55, 3042 (1997).

[48] J. M. Fink, L. Steffen, P. Studer, L. S. Bishop, M. Baur, R. Bianchetti, D. Bozyigit, C. Lang, S. Filipp, P. J. Leek, and A. Wallraff, Quantum-to-Classical Transition in Cavity Quantum Electrodynamics, Phys. Rev. Lett. 105, 163601 (2010).

[49] A. Levy and R. Kosloff, The local approach to quantum transport may violate the second law of thermodynamics, Europhys. Lett. 107, 20004 (2014).

[50] J. T. Stockburger and T. Motz, Thermodynamic deficiencies of some simple Lindblad operators, Fortschr. Phys. 65 (2017).

[51] B. Gardas and S. Deffner, Thermodynamic universality of quantum carnot engines, Phys. Rev. E 92, 042126 (2015).

[52] P. P. Hofer, M. Perarnau-Llobet, L. D. M. Miranda, G. Haack, R. Silva, J. B. Brask, and N. Brunner, Markovian master equations for quantum thermal machines: Local vs global approach (to be published), New J. Phys., arXiv:1707.09211 (2017).

[53] J. O. González, L. A. Correa, G. Nocerino, J. P. Palao, D. Alonso, and G. Adesso, Testing the validity of the local and global gkls master equations on an exactly solvable model, arXiv:1707.09228 (2017).

[54] M. Scala, B. Militello, A. Messina, S. Maniscalco, J. Piilo, and K. Suominen, Cavity losses for the dissipative JaynesCummings Hamiltonian beyond rotating wave approximation, J. Phys. A 40, 14527 (2007).

[55] D. Hu, S.-Y. Huang, J.-Q. Liao, L. Tian, and H.-S. Goan, Quantum coherence in ultrastrong optomechanics, Phys. Rev. A 91, 013812 (2015).
[56] J. Teufel, T. Donner, D. Li, J. Harlow, M. Allman, K. Cicak, A. Sirois, J. D. Whittaker, K. Lehnert, and R. W. Simmonds, Sideband cooling of micromechanical motion to the quantum ground state, Nature 475, 359 (2011).

[57] J. Johansson, P. Nation, and F. Nori, Qutip 2: A python framework for the dynamics of open quantum systems, Comput. Phys. Commun. 184, 1234 (2013).

[58] H. Wichterich, M. J. Henrich, H.-P. Breuer, J. Gemmer, and M. Michel, Modeling heat transport through completely positive maps, Phys. Rev. E 76, 031115 (2007).

[59] T. Feldmann and R. Kosloff, Characteristics of the limit cycle of a reciprocating quantum heat engine, Phys. Rev. E 70, 046110 (2004).

[60] M. P. da Silva, D. Bozyigit, A. Wallraff, and A. Blais, Schemes for the observation of photon correlation functions in circuit QED with linear detectors, Phys. Rev. A 82, 043804 (2010).

[61] J. Klaers, S. Faelt, A. Imamoglu, and E. Togan, Squeezed Thermal Reservoirs as a Resource for a Nano-Mechanical Engine Beyond the Carnot Limit, Phys. Rev. X 7, 031044 (2017).

[62] J. Klatzow, C. Weinzetl, P. M. Ledingham, J. N. Becker, D. J. Saunders, J. Nunn, I. A. Walmsley, R. Uzdin, and E. Poem, Experimental demonstration of quantum effects in the operation of microscopic heat engines, arXiv:1710.08716 (2017).

[63] Y. Zou, Y. Jiang, Y. Mei, X. Guo, and S. Du, Quantum Heat Engine using Electromagnetically Induced Transparency, Phys. Rev. Lett. 119, 050602 (2017).

[64] P. Talkner, E. Lutz, and P. Hänggi, Fluctuation theorems: Work is not an observable, Phys. Rev. E 75, 050102 (2007).

[65] M. Horodecki and J. Oppenheim, Fundamental limitations for quantum and nanoscale thermodynamics, Nat. Commun. 4, 2059 (2013).

[66] P. Skrzypczyk, A. J. Short, and S. Popescu, Work extraction and thermodynamics for individual quantum systems, Nat. Commun. 5, 5185 (2014).

[67] R. Kubo, The fluctuation-dissipation theorem, Rep. Prog. Phys. 29, 255 (1966).

[68] A. Ghosh, C. Latune, L. Davidovich, and G. Kurizki, Catalysis of heat-to-work conversion in quantum machines, Proc. Natl. Acad. Sci. USA 114, 12156 (2017).

[69] J. Z. Bernád and J. M. Torres, Partly invariant steady state of two interacting open quantum systems, Phys. Rev. A 92, 062114 (2015). 